Pacific Journal of Mathematic 


\title{
MACDONALD'S THEOREM FOR QUADRATIC JORDAN ALGEBRAS
}

\author{
ROBERT E. LewAND AND Kevin MCCRIMmon
}

\begin{abstract}
Macdonald's Theorem says that if an identity in three variables $x, y, z$ which is linear in $z$ holds for all special Jordan algebras, it holds for all Jordan algebras. We show this is equivalent to saying the universal quadratic envelope $\mathscr{Q} \mathscr{Q} \mathscr{E}\left(\mathfrak{J}^{(2)}\right)$ of the free Jordan algebra $\mathfrak{\Im}^{(2)}$ on two generators $x, y$ is canonically isomorphic to the universal compound linear envelope $\mathscr{U} \mathscr{C} \mathscr{E}\left(\mathfrak{S}^{(2)}\right)$. We generalize Macdonald's Theorem from the case of linear Jordan algebras over a field of characteristic $\neq 2$ to quadratic Jordan algebras over an arbitrary ring of scalars, at the same time improving on the results in the linear case by presenting $\mathscr{U} \mathscr{Q} \mathscr{E}\left(\mathfrak{S}^{(2)}\right)$ in terms of a finite number of generators and relations. Similarly we generalize Macdonald's Theorem with Inverses concerning identities in $x, x^{-1}, y, y^{-1}, z$. Finally, we prove Shirshov's Theorem that $\Im^{(2)}$ is special.
\end{abstract}

\section{PART I. MACDONALD'S THEOREM.}

1. Free algebras and free products. Throughout this paper $\Phi$ will denote a fixed ring of scalars (= unital commutative, associative ring), and "linear space", "linear map", etc. will always mean linear with respect to $\Phi$.

Recall [4, p. 000] that a unital quadratic algebra $\cong=(\mathfrak{X}, U, 1)$ is a linear space $\mathfrak{X}$ together with a quadratic mapping $x \rightarrow U(x)=U_{x}$ of $\mathfrak{X}$ into $\operatorname{Hom}_{\mathfrak{p}}(\mathfrak{X}, \mathfrak{X})$ and a unit element $1 \in \mathfrak{X}$ satisfying $U_{1} x=x$ and $\{x 1 y\}=$ $\left\{\begin{array}{lll}x & y & 1\end{array}\right\}$ for all $x, y$ (where, as usual, $\left\{\begin{array}{lll}x & y & z\end{array}\right\}=U_{x, z} y=\left\{U_{x+z}-U_{x}-U_{z}\right\} y$ is trilinear). A homomorphism $\varphi: \Omega \rightarrow \tilde{\mathfrak{D}}$ is a linear map satisfying

$$
\varphi(1)=\tilde{1} \quad \varphi\left(U_{x} y\right)=\widetilde{U}_{\varphi(x)} \varphi(y) .
$$

An $i$ deal is a subspace $\Re \subset \mathfrak{Q}$ such that $U_{\mathfrak{R}} \mathfrak{R} \subset \mathfrak{R}, U_{\mathfrak{Q}} \mathfrak{R} \subset \mathfrak{R},\{\mathfrak{R} \mathfrak{Q}\} \subset \Re$.

Given any set $X$ we can construct a free unital quadratic algebra $\mathscr{F} \mathscr{Q}(X)$ on $X$ with an imbedding $i: X \rightarrow \mathscr{F} \mathscr{Q}(X)$ having the following universal property: any (set-theoretic) $\operatorname{map} \varphi: X \rightarrow \mathfrak{Q}$ of $X$ into a unital quadratic algebra $\mathfrak{Q}$ extends uniquely to a homomorphism $\hat{\varphi}: \mathscr{F} \mathscr{Q}(X) \rightarrow \mathfrak{Q}$, i.e., $\varphi=\hat{\varphi} \circ i$. The construction goes as follows $[1$, p. 116]. We recursively define monomials in the elements of $X$, starting with the empty monomial 1 of degree 0 and the monomials $x \in X$ of degree 1 , and using monomials $m, n, p$ of degrees $i, j, k$ to form new monomials $(m ; n)$ of degree $2 i+j$ and $(m, n ; p)=(n, m ; p)$ of degree $i+j+k$; we identify $(1 ; m)$ with $m,(m, n ; 1)$ with $(m, 1 ; n)$, 
and $(m, m ; n)$ with $2(m ; n)$. Then $\mathscr{F} \mathscr{Q}(X)$ is the free module with the monomials as basis and $U$-operator

$$
U_{\sum \alpha_{i} m_{i}} \sum \beta_{j} n_{j}=\sum \alpha_{i}^{2} \beta_{j}\left(m_{i} ; n_{j}\right)+\sum \alpha_{i} \alpha_{j} \beta_{k}\left(m_{i}, m_{j} ; n_{k}\right) .
$$

Thus $(m ; n)=U_{m} n$ and $(m, n ; p)=U_{m, n} p$.

A unital quadratic Jordan algebra is a unital quadratic algebra satisfying the axioms
$(U Q J$ I $) \quad U_{1}=I$
(UQJ II ) $U_{U(x) y}=U_{x} U_{y} U_{x}$
$\left(U Q J \mathrm{II}^{\prime}\right) \quad U_{U(x) y, U(x, z) y}=U_{x} U_{y} U_{x, z}+U_{x, z} U_{y} U_{x}$
$\left(U Q J \mathrm{II}^{\prime \prime}\right) \quad U_{U(x) y, U(z) y}+U_{U(x, z) y}=U_{x} U_{y} U_{z}+U_{z} U_{y} U_{x}+U_{x, z} U_{y} U_{x, z}$
(UQJ III) $\quad U_{x} V_{y, x}=V_{x, y} U_{x}$ and $\left(V_{x, y}=V_{x} V_{y}-U_{x, y}, V_{x}=U_{x, 1}\right)$
$\left(U Q J\right.$ III') $\quad U_{x} V_{y, z}+U_{x, z} V_{y, x}=V_{z, y} U_{x}+V_{x, y} U_{x, z}$.

If $\Re$ denotes the ideal in $\mathscr{F} \mathscr{Q}(X)$ generated by all $U_{U(a) b} c-U_{a} U_{b} U_{a} c$, etc. corresponding to $U Q J$ II, etc. for $a, b, c$ in $\mathscr{F} \mathscr{Q}(X)$ then $\mathscr{F} \mathscr{Q} \mathscr{J}(X)=\mathscr{F} \mathscr{Q}(X) / \Re$ and the map $i: X \rightarrow \mathscr{F} \mathscr{Q} \mathscr{J}(X)$ constitute a free unital quadratic Jordan algebra on $X$ in the sense that they satisfy the universal property that any $\operatorname{map} \varphi: X \rightarrow \Im$ of $X$ into a unital quadratic Jordan algebra factors uniquely through a homomorphism $\hat{\varphi}, \hat{\varphi} \circ i=\varphi$.

Henceforth we will concern ourselves only with unital quadratic Jordan algebras, so we refer to them simply as Jordan algebras. As usual, any Jordan algebra is a homomorphic image (or quotient) of a free one. Also, if $X \subset Y$ then the canonical homomorphisms extending $\varphi: X \rightarrow \mathscr{F} \mathscr{Q} \mathscr{J}(Y)$ and $\psi: Y \rightarrow \mathscr{F} \mathscr{Q} \mathscr{J}(X)$ by $\varphi(x)=i(x)$, $\psi(x)=i(x)$ if $x \in X \subset Y$ and $\psi(y)=0$ if $y \notin X$ have $\psi \circ \varphi=1_{\mathscr{F} \odot \mathscr{f}(X)}$, and the canonical map $\varphi: \mathscr{F} \mathscr{Q} \mathscr{J}(X) \rightarrow \mathscr{F} \mathscr{Q} \mathscr{J}(Y)$ is injective:

$$
\mathscr{F} \mathscr{Q} \mathscr{J}(X) \subset \mathscr{F} \mathscr{Q} \mathscr{J}(Y) \text { if } X \subset Y \text {. }
$$

The free composition [1, p. 113] of Jordan algebras $\Im_{1}, \Im_{2}$ is a Jordan algebra $\mathfrak{\Im}_{1} * \mathfrak{I}_{2}$ together with homomorphisms $\varphi_{i}: \mathfrak{\Im}_{i} \rightarrow \mathfrak{\Im}_{1} * \mathfrak{I}_{2}$ such

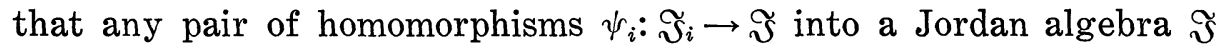
factors uniquely through a homomorphism $\psi: \mathfrak{\Im}_{1} * \Im_{2} \rightarrow \mathfrak{\Im}$ (i.e., $\psi \circ \varphi_{i}=$ $\left.\psi_{i}\right)$. The free composition always exists; if $\Im_{i}=\mathscr{F} \mathscr{Q} \mathscr{J}\left(X_{i}\right) / \Re_{i}$ for $X_{1}, X_{2}$ disjoint sets then $\mathfrak{\Im}_{1} * \Im_{2}=\mathscr{F} \mathscr{Q} \mathscr{J}\left(X_{1} \cup X_{2}\right) / \Re$ for $\Re$ the ideal generated by $\Re_{1}$ and $\Omega_{2}$. In particular, note that if $X, Y$ are disjoint

$$
\mathscr{F} \mathscr{Q} \mathscr{J}(X) * \mathscr{F} \mathscr{Q} \mathscr{J}(Y)=\mathscr{F} \mathscr{Q} \mathscr{J}(X \cup Y) \text {. }
$$

We denote by $\Im[z]$ the free composition $\Im * \mathscr{F} \mathscr{O} \mathscr{J}(z)$, with homomorphisms $\varphi_{1}: \mathfrak{\Im} \rightarrow \Im[z], \varphi_{2}: \mathscr{F} \mathscr{Q} \mathscr{J}(z) \rightarrow \Im[z]$.

THEOREM 1. For any unital quadratic Jordan algebra $\mathfrak{~ t h e ~}$ canonical homomorphism $\mathscr{\mathscr { C }} \mathscr{Q} \mathscr{E}(\Im) \rightarrow \mathscr{C}(\Im \mid \Im[z])$ is an isomorphism. 
We refer to [4] for the basic results about representations, multiplication algebras, and quadratic envelopes. We begin with a preliminary result.

Proposition 1. If $\varphi: \Im \rightarrow \widetilde{\Im}$ is an epimorphism of unital quadratic Jordan algebras then we have an induced homomorphism $\mathscr{L}(\varphi): \mathscr{L C}(\Im) \rightarrow \mathscr{C}(\widetilde{\Im})$ sending $U_{x} \rightarrow \widetilde{U}_{\varphi(x)}$. If $\Re, \widetilde{\Re}$ are subalgebras of $\mathfrak{\Im}, \widetilde{\Im}$ respectively with $\varphi(\Re) \subset \tilde{\Re}$ then by restriction we obtain $a$ homomorphism $\mathscr{l l}(\varphi): \mathscr{L l}(\Re \mid \widetilde{\Im}) \rightarrow \mathscr{L}(\tilde{\Re} \mid \widetilde{\Im})$.

Proof. The only possibility for $\mathscr{C}(\varphi)$ is the map sending $T=$ $\sum U_{x_{1}} \ldots U_{x_{n}}$ in $\mathscr{C}(\widetilde{\Im})$ into $\widetilde{T}=\sum \widetilde{U}_{\varphi\left(x_{1}\right)} \ldots \widetilde{U}_{\varphi\left(x_{n}\right)}$ in $\mathscr{C}(\widetilde{\Im})$. This works if it is well defined, and $T \equiv 0$ implies $0=\varphi(T x)=\widetilde{T}(\varphi(x))$ for all $x$, hence $\widetilde{T}$ kills $\varphi(\Im)=\widetilde{\Im}$ by surjectivity, so $\widetilde{T} \equiv 0$. If $\varphi(\Re) \subset \tilde{\Re}$ then $\mathscr{C}(\varphi)$ maps $\mathscr{C}(\Re \mid \Im)$ into $\mathscr{L}(\tilde{\Re} \mid \widetilde{\Im})$.

Proof of the theorem. The homomorphism $\varphi_{1}: \Im \rightarrow \Im[z]$ induces a unital multiplication specialization $x \rightarrow U_{x}$ of $\mathfrak{\Im}$ in $\mathscr{L}(\Im \mid \Im[z])$, hence a unital quadratic specialization $\mu(x)=U_{x}$, and this factors through the universal specialization $u$ of $\mathscr{Q} \mathscr{Q} \mathscr{E}(\mathfrak{\Im}): \mu=\hat{\mu} \circ u$ for $\hat{\mu}: \mathscr{L} \mathscr{Q} \mathscr{E}(\Im) \rightarrow \mathscr{M}(\Im \mid \Im[z])$ a homomorphism of associative algebras. Clearly $\hat{\mu}$ is surjective, so we need only prove it is injective.

Suppose $m \in \mathscr{U} \mathscr{Q} \mathscr{E}(\Im)$ is in the kernel of $\hat{\mu}$. $\mathscr{C}=\mathscr{U} \mathscr{Q} \mathscr{E}(\Im)$ is a cyclic $\Im$-bimodule with generator $e=u(1)$ relative to the quadratic representation $u_{L}(x)=L_{u(x)}$, and the associated homomorphism $\hat{\mu}_{L}$ of $\mathscr{U} \mathscr{Q} \mathscr{E}(\Im)$ into $\operatorname{Hom}_{\mathscr{Q}}(\mathscr{C}, \mathscr{C})$ is just the left regular representation $a \rightarrow L_{a}$ of $\mathscr{U} \mathscr{Q} \mathscr{E}(\Im)$. We have a homomorphism $\psi$ of $\mathfrak{\Im}[z]$ into the split null extension $\mathfrak{F}=\mathfrak{\Im} \oplus \mathfrak{M}$ synthesizing the homomorphisms $\psi_{1}: \mathfrak{\Im} \rightarrow$ $\Im \subset \mathbb{F}$ and $\psi_{2}: \mathscr{F} \mathscr{Q} \mathscr{J}(z) \rightarrow \mathfrak{M} \subset \mathbb{F}$ by $\psi_{2}(z)=e . \quad \psi$ is an epimorphism since its range contains the generators $\Im$ and $e$ of $\mathfrak{F}$. By Proposition 1 we have a homomorphism $\mathscr{C}(\psi): \mathscr{L}(\Im \mid \Im[z]) \rightarrow \mathscr{C}(\mathfrak{\Im} \mid \mathfrak{F})$, and a restriction homomorphism $\pi: \mathscr{C}(\mathfrak{J} \mid \mathfrak{F}) \rightarrow \mathscr{M}(\mathfrak{W} \mid \mathfrak{M})$ since $\mathfrak{M}$ is a $\mathfrak{~}$ bimodule. The resultant homomorphism $\pi \circ \mathscr{l}(\psi) \circ \hat{\mu}: \mathscr{C} \mathscr{Q} \mathscr{E}(\Im) \rightarrow$ $\mathscr{C}(\mathfrak{S} \mid \mathfrak{M})$ coincides with $\hat{u}_{L}$ since on the generators $u(x)$ of $\mathscr{U} \mathscr{Q} \mathscr{E}(\mathfrak{F})$ we have $\pi\left(\mathscr{L}(\psi)(\hat{\mu}(u(x)))=\pi\left(\mathscr{C l}\left(\psi^{\prime}\right)\left(u_{x} \mid \mathfrak{\Im}[z]\right)\right)=\pi\left(U_{x} \mid[\mathfrak{s})=u_{L}(x)\right.\right.$. Thus $0=\{\pi \circ \mathscr{C l}(\psi)\}\left(\hat{\mu}^{\prime}(m)\right)=\widehat{u}_{L}(m)=L_{m}$ implies $m=L_{m} e=0$, and $\hat{\mu}$ is injective.

Taking $\mathfrak{\Im}$ to be the free Jordan algebra $\mathfrak{\Im}^{(n-1)}=\mathscr{F} \mathscr{Q} \mathscr{J}\left(x_{1}\right.$, $\left.\cdots, x_{n-1}\right)$ on $n-1$ generators, and noting $\mathfrak{\Im}^{(n-1)}[z]=\Im^{(n-1)} * \mathscr{F} \mathscr{Q} \mathscr{J}(z) \cong$ $\mathscr{F} \mathscr{Q} \mathcal{J}\left(x_{1}, \cdots, x_{n-1}\right) * \mathscr{F} \mathscr{Q} \mathcal{J}\left(x_{n}\right)=\mathscr{F} \mathscr{Q} \mathscr{J}\left(x_{1}, \cdots, x_{n}\right)=\mathfrak{F}^{(n)}$ by $(2)$, we have

COROLLARY. The universal unital quadratic envelope for the free 
unital quadratic Jordan algebra $\mathfrak{\Im}^{(n-1)}$ on $n-1$ generators is isomorphic to the multiplication algebra of $\mathfrak{\Im}^{(n-1)}$ on $\mathfrak{\Im}^{(n)}$ :

$$
\mathscr{U} \mathscr{Q} \mathscr{E}\left(\mathfrak{\Im}^{(n-1)}\right) \cong \mathscr{M}\left(\mathfrak{\Im}^{(n-1)} \mid \mathfrak{F}^{(n)}\right) \text {. }
$$

The free special Jordan algebra $\mathscr{F} \mathscr{S} \mathscr{J}(X)$ on a set $X$ is easier to construct: it is the (Jordan) subalgebra generated by the elements $x \in X$ of the free associative algebra $\mathscr{F} \mathscr{A}(X)$ on $X$. It has the universal property that any map $\varphi: X \rightarrow \Im_{s}$ of $X$ into a special Jordan algebra $\mathfrak{\Im}_{s}$ extends uniquely to a Jordan homomorphism $\hat{\varphi}: \mathscr{F} \mathscr{S} \mathscr{J}(X) \rightarrow \mathfrak{\Im}_{s}$. If we denote the free special Jordan algebra on $n$ generators by $\Im_{s}^{(n)}$, and similarly for the free associative algebra $\mathfrak{Y}^{(n)}$, then

$$
\mathfrak{\Im}_{s}^{(n-1)} \subset \mathfrak{J}_{s}^{(n)} \subset \mathfrak{Y}^{(n)+} .
$$

THEOREM 2. The universal unital compound linear envelope of the free special unital quadratic Jordan algebra $\mathfrak{\Im}_{s}^{(n-1)}$ on $n-1$ generators is canonically isomorphic to the multiplication algebra of $\Im_{s}^{(n-1)}$ on $\mathfrak{\Im}_{s}^{(n)}$ :

$$
\mathscr{U} \mathscr{C} \mathscr{E}\left(\Im_{s}^{(n-1)}\right) \cong \mathscr{C}\left(\Im_{s}^{(n-1)} \mid \Im_{s}^{(n)}\right)
$$

Proof. We know $\left[4\right.$, p. 000] that $\mathscr{U} \mathscr{C} \mathscr{E}\left(\mathfrak{\Im}_{s}^{(n-1)}\right)$ is the subalgebra of $\mathfrak{Y}^{(n-1)} \otimes \mathfrak{P}^{(n-1)}$ generated by all $x \otimes x$ for $x \in \Im_{s}^{(n-1)} \subset \mathfrak{X}^{(n-1)}$ since the universal unital linear envelope for $\mathfrak{\Im}_{s}^{(n-1)}$ is $\mathscr{U} \mathscr{L} \mathscr{E}\left(\mathfrak{\Im}_{s}^{(n-1)}\right)=\mathfrak{U}^{(n-1)}$. Now $\mathfrak{A}^{(n-1)} \otimes \mathfrak{A}^{(n-1)}$ is isomorphic to the (associative). multiplication algebra $\mathscr{C}\left(\mathfrak{U}^{(n-1)} \mid \mathfrak{A}^{(n)}\right)=L_{\mathfrak{O}^{(n-1)}} R_{\mathfrak{X}^{(n-1)}} \quad$ under $\quad a \otimes b \rightarrow L_{a} R_{b^{*}} \quad$ (*the reversal involution on $\left.\mathfrak{2}^{(n-1)}\right)$, and the subalgebra generated by all $L_{x} R_{x}=U_{x}$ for $x=x^{*}$ in $\Im_{s}^{(n-1)}$ is just the (Jordan) multiplication algebra $\mathscr{M}\left(\mathfrak{\Im}_{s}^{(n-1)} \mid \mathfrak{U}^{(n)+}\right)$. Now $\mathfrak{\Im}_{s}^{(n)}$ is invariant under $\mathfrak{\Im}_{s}^{(n-1)}$, and the restriction epimorphism $\mathscr{L}\left(\mathfrak{\Im}_{s}^{(n-1)} \mid \mathfrak{U}^{(n)+}\right) \rightarrow \mathscr{M}\left(\mathfrak{\Im}_{s}^{(n-1)} \mid \mathfrak{\Im}_{s}^{(n)}\right)$ is an isomorphism: for any $a \in \mathfrak{Y}^{(n)+}$ there is a homomorphism $\varphi_{a}: \mathfrak{J}_{s}^{(n)} \rightarrow \mathfrak{\mathfrak { U } ^ { ( n ) + }}$ fixing $\mathfrak{\Im}_{s}^{(n-1)}$ but sending $x_{n}$ into $a$, so if $T \in \mathscr{C}\left(\mathfrak{\Im}_{s}^{(n-1)} \mid \mathfrak{U}^{(n)+}\right)$ restricts to zero on $\Im_{s}^{(n)}\left(T\left(\Im_{s}^{(n)}\right)=0\right)$ then $0=\varphi_{a}\left(T\left(x_{n}\right)\right)=T\left(\varphi_{a}\left(x_{n}\right)\right)=T a$, so $T$ kills $\mathfrak{2}^{(n)+}$, and $T$ is zero as a transformation on $\mathfrak{2}^{(n)+}$. Thus

$$
\mathscr{C} \mathscr{C} \mathscr{C}\left(\Im_{s}^{(n-1)}\right) \cong \mathscr{C}\left(\mathfrak{\Im}_{s}^{(n-1)} \mid \mathfrak{H}^{(n)}\right) \cong \mathscr{C}\left(\mathfrak{\Im}_{s}^{(n-1)} \mid \mathfrak{\Im}_{s}^{(n)}\right)
$$

2. Equivalent forms of Macdonald's theorem. Let $\Im^{(n)}$ and $\mathfrak{\Im}_{s}^{(n)}$ denote the free and free special Jordan algebras on $n$ generators $x_{1}, \cdots, x_{n}$. By (1) we may assume $\Im^{(n)} \subset \Im^{(m)}$ if $n \leqq m$. We have the canonical epimorphism

$$
\nu^{(n)}: \Im^{(n)} \longrightarrow \mathfrak{\Im}_{s}^{(n)}
$$

determined by $\nu^{(n)}\left(x_{i}\right)=x_{i}(1 \leqq i \leqq n)$. The kernel of $\nu^{(n)}$ is an ideal 


$$
\AA^{(n)}=\operatorname{Ker} \nu^{(n)}
$$

in $\Im^{(n)}$. We let $\mathfrak{Z}^{(n)}, \mathfrak{Z}_{s}^{(n)}$ denote the subspaces of $\mathfrak{\Im}^{(n)}$, $\mathfrak{\Im}_{s}^{(n)}$ respectively consisting of elements homogeneous of degree one in the variable $x_{n}$. (For $n=3$ we write $x, y, z$ for $x_{1}, x_{2}, x_{3}$, so $3^{(3)}, 3_{s}^{(3)}$ are homogeneous of degree one in $z$ ). The original form [3] of Macdonald's Theorem was that

(I) $\mathscr{\Re}^{(n)} \cap 3^{(n)}=0$

when $n=3$. Since $\mathcal{V}^{(n)}\left(3^{(n)}\right)=3_{s}^{(n)}$, this is equivalent to

(II) $\mathcal{V}^{(n)}: 3^{(n)} \rightarrow 3_{s}^{(n)}$ is bijective.

These two forms say that if some identity in $x_{1}, \ldots, x_{n}$ which is linear in $x_{n}$ (corresponding to an element of $3^{(n)}$ ) holds in all special algebras (its image under $\nu^{(n)}$ is zero) then it holds in all Jordan algebras (it is zero in $3^{(n)}$ ). This is the most practical form of Macdonald's result. Since identities are harder to prove in the case of quadratic Jordan algebras than for linear Jordan algebras, an algorithm like this is a great laborsaving device.

The multiplication algebra $\mathscr{C}\left(\mathfrak{\Im}^{(n-1)} \mid \mathfrak{\Im}^{(n)}\right)$ of $\mathfrak{\Im}^{(n-1)}$ on $\mathfrak{\Im}^{(n)}$ is generated by the transformations $I, V_{x_{i}}, U_{x_{i}}, U_{x_{i}, x_{j}}$ for $1 \leqq i, j \leqq n$ by [4, p. 000]. By Proposition 1 the epimorphism $\nu^{(n)}: \mathfrak{\Im}^{(n)} \rightarrow \mathfrak{\Im}_{s}^{(n)}$ induces an epimorphism $\mathscr{C}\left(\mathcal{\nu}^{(n)}\right): \mathscr{C}\left(\mathfrak{\Im}^{(n-1)} \mid \mathfrak{\Im}^{(n)}\right) \rightarrow \mathscr{C}\left(\mathfrak{\Im}_{s}^{(n-1)} \mid \mathfrak{\Im}_{s}^{(n)}\right)$. We have a linear map $z: \mathscr{C}\left(\Im^{(n-1)} \mid \Im^{(n)}\right) \rightarrow 3^{(n)}$ sending $T \rightarrow T\left(x_{n}\right)$. This is surjective by the definition of $3^{(n)}$ as the space spanned by all Jordan products of $x_{1}, \cdots, x_{n}$ which are linear in $x_{n}$, and it is injective since if $T\left(x_{n}\right)=0$ then $T(x)=0$ for any $x$ in $\mathfrak{J}^{(n)}$ by the usual argument (there is an endomorphism $\varphi$ of $\Im^{(n)}$ fixing $x_{i}$ for $1 \leqq i \leqq n-1$ but sending $x_{n}$ to $x$, so $\left.0=\varphi\left(T\left(x_{n}\right)\right)=T\left(\varphi\left(x_{n}\right)\right)=T x\right)$, and $T=0$ as a transformation. We have a similar bijection $z_{s}: \mathscr{L}\left(\mathfrak{J}_{s}^{(n-1)} \mid \mathfrak{\Im}_{s}^{(n)}\right) \rightarrow \mathfrak{\Im}_{s}^{(n)}$, and since $z_{s} \circ \mathscr{C}\left(\boldsymbol{\nu}^{(n)}\right)=\nu^{(n)} \circ z$ we see (II) is equivalent to

(III) $\mathscr{L}\left(\mathcal{\nu}^{(n)}\right): \mathscr{L}\left(\Im^{(n-1)} \mid \Im^{(n)}\right) \rightarrow \mathscr{L}\left(\Im_{s}^{(n-1)} \mid \Im_{s}^{(n)}\right)$ is an isomorphism. This formulation is due to N. Jacobson [2, p. 47].

We have already seen (in the corollary to Theorem 1 and in Theorem 2) that $\mathscr{C}\left(\mathfrak{\Im}^{(n-1)} \mid \mathfrak{\Im}^{(n)}\right)$ is canonically isomorphic to the universal envelope $\mathscr{U} \mathscr{Q} \mathscr{E}\left(\Im^{(n-1)}\right)$ and $\mathscr{C}\left(\Im_{s}^{(n-1)} \mid \Im_{s}^{(n)}\right)$ to the universal compound linear envelope $\mathscr{Q} \mathscr{C} \mathscr{E}\left(\mathfrak{\Im}_{s}^{(n-1)}\right)$ of $\mathfrak{\Im}_{s}^{(n-1)}$. Thus (III) leads to the formulation

(IV) the canonical homomorphism $\mathscr{Q} \mathscr{Q} \mathscr{E}\left(\mathfrak{S}^{(n-1)}\right) \rightarrow \mathscr{W} \mathscr{C} \mathscr{E}\left(\mathfrak{\Im}_{s}^{(n-1)}\right)$ is an isomorphism.

Since it is easily checked that the universal linear envelope of both the

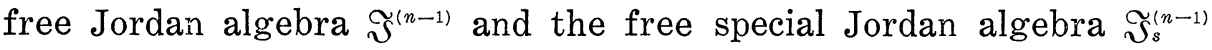
is the free associative algebra $\mathfrak{Y}^{(n-1)}$, and since [4, p. 000] for an arbi-

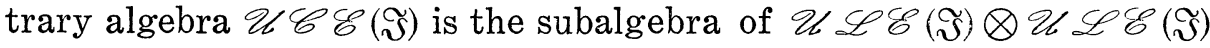
generated by all $l(x) \otimes l(x)$ for $l: \mathfrak{\Im} \rightarrow \mathscr{U} \mathscr{L} \mathscr{E}(\mathfrak{\Im})$ the universal linear specialization, we see $\mathscr{\mathscr { C }} \mathscr{C} \mathscr{E}\left(\mathfrak{\Im}^{(n-1)}\right)=\mathscr{U} \mathscr{C} \mathscr{E}\left(\Im_{s}^{(n-1)}\right)$. Thus (IV) is 
equivalent to $\mathscr{\mathscr { C }} \mathscr{Q} \mathscr{E}\left(\mathfrak{\Im}^{(n-1)}\right) \rightarrow \mathscr{U} \mathscr{C} \mathscr{E}\left(\mathfrak{\Im}^{(n-1)}\right)$ being an isomorphism, so by $[4$, p. 000] to

(V) all unital quadratic representations of the algebra $\Im^{(n-1)}$ are special.

So far we have established.

EquivalenCE THEOREM. The following conditions are equivalent for a given $n$ :

(I ) $\Omega^{(n)} \cap 3^{(n)}=0$

(II) $\nu^{(n)}: 3^{(n)} \rightarrow 3_{s}^{(n)}$ is bijective

(III) $\mathscr{L}\left(\mathcal{\nu}^{(n)}\right): \mathscr{L}\left(\Im^{(n-1)} \mid \mathfrak{\Im}^{(n)}\right) \rightarrow \mathscr{L}\left(\Im_{s}^{(n-1)} \mid \mathfrak{\Im}_{s}^{(n)}\right)$ is an isomorphism

(IV) the canonical homomorphism $\mathscr{\mathscr { C }} \mathscr{Q} \mathscr{E}\left(\Im^{(n-1)}\right) \rightarrow \mathscr{Q} \mathscr{C} \mathscr{E}\left(\mathfrak{\Im}_{s}^{(n-1)}\right)$ is an isomorphism

(V) all unital quadratic representations of the algebra $\mathfrak{\Im}^{(n-1)}$ are special

where $\mathfrak{\Im}^{(m)}, \Im_{s}^{(m)}$ denote the free and free special unital quadratic Jordan algebras on the generators $x_{1}, \cdots, x_{m}, \Omega^{(m)}$ the kernel of the canonical homomorphism $\nu^{(m)}: \Im^{(m)} \rightarrow \Im_{s}^{(m)}$, and $3^{(m)}, 3_{s}^{(m)}$ the subspaces of $\Im^{(m)}, \Im_{s}^{(m)}$ respectively consisting of elements linear in the variable $x_{m}$.

Macdonald's Theorem says that these (equivalent) conditions hold for $n \leqq 3$ (an example due to Glennie [2, p. 51] shows they fail for $n \geqq 4)$. We choose the formulations I and II as the most convenient in practice.

MACDONALD's Theorem (Practical Form). If an identity in the variables $x, y, z$ is linear in $z$ and holds in all special quadratic Jordan algebras then it holds in all quadratic Jordan algebras. ${ }^{1}$

Next we turn to the problem of finding a presentation of $\mathscr{U} \mathscr{Q} \mathscr{E}\left(\mathfrak{S}^{(2)}\right)$ by generators and relations. Let $\mathfrak{F}$ denote the free associative algebra on the five generators $a, b, c, d, e$. We have a homomorphism

$$
\mathfrak{F} \longrightarrow \mathscr{U} \mathscr{Q} \mathscr{E}\left(\Im^{(2)}\right)
$$

mapping $a \rightarrow v(x), b \rightarrow u(x), c \rightarrow v(y), d \rightarrow u(y), e \rightarrow u(x, y)$. Since 1, $v(x)$, $u(x), v(y), u(y), u(x, y)$ generate $\mathscr{U} \mathscr{Q} \mathscr{E}\left(\mathfrak{F}^{(2)}\right)$ if $1, x, y$ generate $\mathfrak{F}^{(2)}$ $[4$, p. 000] we see this is an epimorphism.

As special cases or linearizations of the axiom UQS III

$$
u(z) v(w, z)=v(z, w) u(z)
$$

for quadratic specializations $[4, \mathrm{p}$. 000] we have the following rela-

\footnotetext{
1 This result has been obtained independently by Professor John Faulkner.
} 
tions among the generators of $\mathscr{U} \mathscr{Q} \mathscr{E}\left(\mathfrak{\Im}^{(2)}\right)$ :

( 3 )
( i ) $u(x) v(x)=v(x) u(x), u(y) v(y)=v(y) u(y)$
(ii) $u(x) v(y)+u(x, y) v(x)=v(y) u(x)+v(x) u(x, y)$, $u(y) v(x)+u(y, x) v(y)=v(x) u(y)+v(y) u(y, x)$
(iii) $u(x) v(y, x)=v(x, y) u(x), \quad u(y) v(x, y)=v(y, x) u(y)$
(iv) $u(x) v(y, y)+u(x, y) v(y, x)=v(y, y) u(x)+v(x, y) u(x, y)$, $u(y) v(x, x)+u(y, x) v(x, y)=v(x, x) u(y)+v(y, x) u(y, x)$
(v) $u(x) v\left(y^{2}, x\right)=v\left(x, y^{2}\right) u(x), \quad u(y) v\left(x^{2}, y\right)=v\left(y, x^{2}\right) u(y)$
(vi) $u(x) v\left(y^{3}, x\right)=v\left(x, y^{3}\right) u(x), \quad u(y) v\left(x^{3}, y\right)=v\left(y, x^{3}\right) u(y)$.

Here

$$
\begin{aligned}
v(z, w) & =v(z) v(w)-u(z, w) \\
\left.v^{\prime} z^{2}\right) & =v(z, z)=v(z)^{2}-2 u(z) \\
v\left(z^{3}\right) & =v(z)^{3}-3 v(z) u(z) \\
u\left(z^{2}, w\right) & =u(z, w) v(z)-v(w) u(z)=v(z) u(w, z)-u(z) v(w) \\
u\left(z^{3}, w\right) & =u\left(z^{2}, w\right) v(z)-u(z, w) u(z)=v(z) u\left(w, z^{2}\right)-u(z) u(z, w) .
\end{aligned}
$$

These correspond to the following relations among the generators:

(R1) $a b=b a, c d=d c$

(R2) $a e+c b=e a+b c, c e+a d=e c+d a$

(R3) $b\{c a-e\}=\{a c-e\} b, d\{a c-e\}=\{c a-e\} d$

(R4) $b\left\{c^{2}-2 d\right\}+e\{c a-e\}=\left\{c^{2}-2 d\right\} b+\{a c-e\} e$,

$$
d\left\{a^{2}-2 b\right\}+e\{a c-e\}=\left\{a^{2}-2 b\right\} d+\{c a-e\} e
$$

(R5) $b\left\{\left(c^{2}-2 d\right) a-(c e-d a)\right\}=\left\{a\left(c^{2}-2 d\right)-(e c-a d)\right\} b$

$$
d\left\{\left(a^{2}-2 b\right) c-(a e-b c)\right\}=\left\{c\left(a^{2}-2 b\right)-(e a-c b)\right\} d
$$

(R6) $b\left\{\left(c^{3}-3 c d\right) a-c(c e-d a)+d e\right\}=$

$$
\begin{gathered}
\left\{a\left(c^{3}-3 c d\right)-(e c-a d) c+e d\right\} b, \\
\left.d\left\{a^{3}-3 a b\right) c-a(a e-b c)+b e\right\}=
\end{gathered}
$$$$
\left\{c\left(a^{3}-3 a b\right)-(e a-c b) a+e b\right\} d \text {. }
$$

If $\Re$ denotes the ideal in $\mathfrak{F}$ generated by the relations $\mathrm{R} 1-6$ (i.e., by the elements $a b-b a, a e+c b-e a-b c$, etc.) then we have an induced epimorphism

$$
\sigma: \mathfrak{F} / \Re \longrightarrow \mathscr{Q} \mathscr{Q} \mathscr{E}\left(\mathfrak{\Im}^{(2)}\right) \text {. }
$$

Our proof of Macdonald's Theorem will have as a consequence the fact that $\sigma$ is an isomorphism. Thus $\sigma$ affords a presentation of $\mathscr{U} \mathscr{Q} \mathscr{E}\left(\mathfrak{\Im}^{(2)}\right)$ by means of a finite number of generators and relations.

Presentation THEOREM. The universal unital quadratic envelope $\mathscr{U} \mathscr{Q} \mathscr{E}\left(\mathfrak{\Im}^{(2)}\right)$ of the free unital quadratic Jordan algebra $\mathfrak{\Im}^{(2)}$ on two generators $x, y$ is given abstractly by the six generators 
1, $v(x), u(x), v(y), u(y), u(x, y)$ and the six pairs of relations (3. i-vi).

That these relations are enough to characterize the quadratic envelope again indicates the importance of the axiom $U Q J$ III for Jordan algebras.

3. The crucial lemma. The following lemma is crucial to our proof (and also the proofs using an infinite number of generators and relations [2], [3], [5]). By abuse of notation we continue to denote the images of $a, b, c, d, e$ in the quotient $\mathfrak{F} / \mathscr{\Re}$ by the same symbols.

LEMma 1. There exist elements $a_{k}, b_{k}, c_{k}, d_{k}, e_{k, l}(k, l \geqq 0)$ in $\Im / \Re$ satisfying the relations

(i ) $\quad a_{0}=c_{0}=e_{0,0}=2, b_{0}=d_{0}=1$

$$
a_{1}=e_{1,0}=a, c_{1}=e_{0,1}=c, b_{1}=b, d_{1}=d, e_{1,1}=e
$$

(ii) $b_{i} b_{j}=b_{i+j}, d_{i} d_{j}=d_{i+j}$,

(iii) $e_{2 i+j, k}=a_{i} e_{i+j, k}-b_{i} e_{j, k}=e_{i+j, k} a_{i}-e_{j, k} b_{i}$,

(iv) $a_{i+j} e_{i, k}+a_{i} e_{i+j, k}=b_{i} a_{j} c_{k}+2 e_{2 i+j, k}$

$$
c_{i+j} e_{k, i}+c_{i} e_{k, i+j}=d_{i} c_{j} a_{k}+2 e_{k, 2 i+j} \text {. }
$$

Proof. The first step is to define the $e_{i, j}$. We can use the recursion relations

$$
\begin{aligned}
e_{0,0} & =2 e_{1,0}=a e_{0,1}=c e_{1,1}=e \\
e_{i+2, j} & =a e_{i+1, j}-b e_{i, j}=e_{i+1, j} a-e_{i, j} b \\
e_{i, j+2} & =c e_{i, j+1}-d e_{i, j}=e_{i, j+1} c-e_{i, j} d
\end{aligned}
$$

as long as these are consistent; that is, before we can define $e_{i, j}$ by the above we must know

(a) $a e_{i-1, j}-b e_{i-2, j}=e_{i-1, j} a-e_{i-2, j} b(i \geqq 2)$

(b) $a e_{i-1, j}-b e_{i-2, j}=e_{i, j-1} c-e_{i, j-2} d(i, j \geqq 2)$

and their duals (obtained by replacing $a, b, c, d, e_{k, l}$ with $c, d, a, b, e_{l, k}$ ). We induct on $i+j$, assuming $e_{i^{\prime}, j^{\prime}}$ is given by the common value of (a) and (b) for $i^{\prime}+j^{\prime}<i+j$. Then (b) follows immediately:

$$
\begin{aligned}
& a e_{i-1, j}-b e_{i-2, j}=a\left\{e_{i-1, j-1} c-e_{i-1, j-2} d\right\}-b\left\{e_{i-2, j-1} c-e_{i-2, j-2} d\right\} \\
& \quad=\left\{a e_{i-1, j-1}-b e_{i-2, j-1}\right\} c-\left\{a e_{i-1, j-2}-b e_{i-2, j-2}\right\} d \\
& \quad=e_{i, j-1} c-e_{i, j-2} d .
\end{aligned}
$$

From this we see that for $i, j \geqq 2$ the validity of (a) and its dual 
for a given $(i, j)$ implies its validity for $(j, i)$. In particular, we need only consider (a) for $i \geqq j$.

In (a), if $i \geqq 4$ we have

$$
\begin{aligned}
& a e_{i-1, j}-b e_{i-2, j}=a\left\{e_{i-2, j} a-e_{i-3, j} b\right\}-b\left\{e_{i-3, j} a-e_{i-4, j} b\right\} \\
& \quad=\left\{a e_{i-2, j}-b e_{i-3, j}\right\} a-\left\{a e_{i-3, j}-b e_{i-4, j}\right\} b \\
& \quad=e_{i-1, j} a-e_{i-2, j} b
\end{aligned}
$$

by induction. Thus only the cases $2 \leqq i \leqq 3,0 \leqq j \leqq i$ remain. We can easily dispose of the case $j=0$ since the recursion relation shows $e_{i, 0}$ is a polynomial in the commuting variables $a$ and $b$ (using R1). When $j=1$ the relation for $i=2$ becomes $a e-b c=e a-c b$ (since $\left.e_{0,1}=c, e_{1,1}=e\right)$, which is just R2, while for $i=3$ it becomes $a\{e a-c b\}-b e=\{a e-b c\} a-e b$ (since $\left.e_{2,1}=a e-b c=e a-c b\right)$, which follows from R3 after cancelling aea from both sides. This leaves the three cases $2 \leqq j \leqq i \leqq 3$.

The case $i=j=2$ is $a\{c e-d a\}-b\left\{c^{2}-2 d\right\}=\{e c-a d\} a-\left\{c^{2}-2 d\right\} b$ since $e_{0,2}=c^{2}-2 d$; cancelling $-a d a$ and subtracting $e^{2}$ from both sides results in $\mathrm{R} 4$.

The case $i=3, j=2$ is $a\left\{e_{1,2} a-e_{0,2} b\right\}-b\{c e-d a\}=\left\{a e_{1,2}-b e_{0,2}\right\} a-$ $\{e c-a d\} b$; upon cancelling $a e_{1,2} a$ from both sides this becomes R5.

The case $i=j=3$ is $a\left\{e_{1,3} a-e_{0,3} b\right\}-b e_{1,3}=\left\{a e_{1,3}-b e_{0,3}\right\} a-e_{1,3} b$, which becomes R6 upon cancelling $a e_{1,3} a$ from both sides since $e_{0,3}=$ $c^{3}-3 c d$ and $e_{1,3}=c e_{1,2}-d e_{1,1}=e_{1,2} c-e_{1,1} d$.

Once we have defined the $e_{i},{ }_{j}$ we set

$$
a_{k}=e_{k, 0} \quad b_{k}=b^{k} \quad c_{k}=e_{0, k} \quad d_{k}=d^{k} .
$$

The relations (i), (ii) are immediate consequences of our definitions (and the axiom that $a$ commutes with $b, c$ commutes with $d$ ), as are the cases $i=0,1$ of (iii). If (iii) holds for a given $i \geqq 1$ (and all $j, k)$ it holds for $i+1$ since

$$
\begin{aligned}
& a_{i+1} e_{i+1+j, k}-b_{i+1} e_{j, k} \\
&=\left\{a a_{i}-b a_{i-1} e_{i+j+1, k}-b_{i} b e_{j, k}\right. \text { (by definition) } \\
&= a\left\{a_{i} e_{i+j+1, k}\right\}-b_{i}\left\{b e_{j, k}\right\}-b\left\{a_{i-1} e_{i+j+1, k}\right\} \\
&= a\left\{e_{2 i+j+1, k}+b_{i} e_{j+1, k}\right\}-b_{i}\left\{a e_{j+1, k}-e_{j+2, k}\right\}-b\left\{b_{i-1} e_{j+2, k}+e_{2 i+j, k}\right\} \\
&(\text { by the induction hypothesis for } i, 1, i-1) \\
&= a e_{2 i+j+1, k}-b e_{2 i+j, k} \text { (by (ii)) } \\
&= e_{2 i+2+j, k} \text { (by definition) }
\end{aligned}
$$

and similarly for the other parts of (iii). For (iv) we again induct on $i+j$, and we can assume $i, j \geqq 1$ since the result holds trivially for $i=0$ or $j=0$. Then 


$$
\begin{aligned}
a_{i+j} e_{i, k} & +a_{i} e_{i+j, k}=\left\{a a_{i+j-1}-b a_{i+j-2}\right\} e_{i, k} \\
& +a_{i}\left\{a e_{i+j-1, k}-b e_{i+j-2, k}\right\} \text { (by definition) } \\
= & a\left\{a_{i+j-1} e_{i, k}+a_{i} e_{i+j-1, k}\right\}-b\left\{a_{i+j-2} e_{i, k}+a_{i} e_{i+j-2, k}\right\}
\end{aligned}
$$

where if $j=1$ this becomes

$$
\begin{aligned}
& =a\left\{a_{i} e_{i, k}+a_{i} e_{i, k}\right\}-b\left\{a_{i-1} e_{i, k}+a_{i} e_{i-1, k}\right\} \\
& =2 a a_{i} e_{i, k}-b\left\{b_{i-1} a_{1} c_{k}+2 e_{2 i-1, k}\right\} \text { (by induction) } \\
& =2 a\left\{e_{2 i, k}+b_{i} c_{k}\right\}-b_{i} a c_{k}-2\left\{a e_{2 i, k}-e_{2 i+1, k}\right\} \text { (by iii) } \\
& =b_{i} a_{1} c_{k}+2 e_{2 i+1, k}
\end{aligned}
$$

while if $j \geqq 2$ it becomes by induction

$$
\begin{aligned}
& =a\left\{b_{i} a_{j-1} c_{k}+2 e_{2 i+j-1, k}\right\}-b\left\{b_{i} a_{j-2} c_{k}+2 e_{2 i+j-2, k}\right\} \\
& =b_{i}\left\{a a_{j-1}-b a_{j-2}\right\} c_{k}+2\left\{a e_{2 i+j-1, k}-b e_{2 i+j-2, k}\right\} \\
& =b_{i} a_{j} c_{k}+2 e_{2 i+j, k} .
\end{aligned}
$$

This completes the proof of the lemma.

4. Proof of the theorems. The proof now proceeds along the lines of the proof in [5]. We have surjective homomorphisms $\sigma: \mathfrak{F} \mid \mathfrak{A} \rightarrow \mathscr{U} \mathscr{Q} \mathscr{E}\left(\mathfrak{J}^{(2)}\right)$ and $\nu: \mathscr{U} \mathscr{Q} \mathscr{E}\left(\mathfrak{J}^{(2)}\right) \rightarrow \mathscr{C} \mathscr{C} \mathscr{E}\left(\mathfrak{\Im}^{(2)}\right)$, and a linear mapping $\tau: \mathscr{C} \mathscr{C} \mathscr{E}\left(\mathfrak{S}^{(2)}\right) \rightarrow 3_{s}^{(3)}$ by $T \rightarrow T(z)$ (writing $x, y, z$ in place of $x_{1}, x_{2}, x_{3}$ ). If $\mu=\tau \circ \nu \circ \sigma$ is injective so is each of the factors (since $\sigma, \nu$ are surjective); then $\sigma$ is bijective, proving the Presentation Theorem, and $\nu$ is bijective, proving Macdonald's Theorem. We prove $\mu$ is injective by exhibiting a spanning set $f(p), f(p, q)(p \neq q$ monomials in $\mathfrak{A}^{(2)}$ ) for $\mathfrak{F} /$ whose image under $\mu$ is independent in $\mathfrak{Z}_{s}^{(3)}$ (hence both sets are bases, and $\mu$ takes one basis onto the other),

$$
\mu(f(p))=f_{s}(p)=p z p^{*}, \quad \mu(f(p, q))=f_{s}(p, q)=p z q^{*}+q z p^{*} .
$$

It is clear that these latter are independent in $\mathfrak{2}^{(3)}$, and are *-symmetric (* the reversal involution in $\mathfrak{2}^{(3)}$ ); if (5) holds then the $f_{s}(p), f_{s}(p, q)$ will actually be Jordan elements and thus a basis for $3_{s}^{(3)}$. Thus we need only choose pre-images $f(p), f(p, q)$ of $f_{s}(p)$, $f_{s}(p, q)$ in such a way that they span $\widetilde{F} / \Re$; therefore it is enough if they contain

$$
f(1)=1
$$

and satisfy

$$
f(p, p)=2 f(p)
$$

and are invariant under left multiplication by $a_{k}, b_{k}, c_{k}, d_{k}, e_{k, l}$ (hence by the generators $a, b, c, d, e$ ) 
(07) (iii) $e_{k, l} f(p)=f\left(x^{k} p, y^{l} p\right)$

(iv) $a_{k} f(p)=f\left(x^{k} p, p\right)$

(v) $c_{k} f(p)=f\left(y^{k} p, p\right)$

(i ) $b_{k} f(p, q)=f\left(x^{k} p, x^{k} q\right)$

(ii) $d_{k} f(p, q)=f\left(y^{k} p, y^{k} q\right)$

(7) (iii) $e_{k, l} f(p, q)=f\left(x^{k} p, y^{l} q\right)+f\left(y^{l} p, x^{k} q\right)$

(iv) $\quad a_{k} f(p, q)=f\left(x^{k} p, q\right)+f\left(p, x^{k} q\right)$

(v) $c_{k} f(p, q)=f\left(y^{k} p, q\right)+f\left(p, y^{k} q\right)$.

(Note that the right sides of (7) may involve terms of the form $f(p, p)$, which are not a priori spanned by the $f(p)$ and $f(p, q)$ for $p \neq q$; this is why we need (6)).

We define $f(p), f(p, q)$ inductively as follows. We divide the collection of monomials $p$ in $\mathfrak{2}^{(2)}$ into sets $X_{n}, Y_{n}$ depending on their height $n$ and whether they begin with an $x$ or a $y$ :

$$
\begin{aligned}
X_{0}=Y_{0} & =Z_{0}=\{1\}, \quad X_{n+1}=\left\{x^{i} r \mid i \neq 0, r \in Y_{n}\right\}, \\
Y_{n+1} & =\left\{y^{i} r \mid i \neq 0, r \in X_{n}\right\} .
\end{aligned}
$$

We define $f(p)$ on $X_{0}=Y_{0}=Z_{0}$ by

(0D.0)

$$
f(1)=1 \text {, }
$$

on $Y_{n+1}$ by

(0D.1)

$$
f\left(x^{i} r\right)=b_{i} f(r) \quad\left(i \neq 0, r \in Y_{n}\right),
$$

on $X_{n+1}$ by

(0D.2)

$$
f\left(y^{i} r\right)=d_{i} f(r) \quad\left(i \neq 0, r \in X_{n}\right) .
$$

For the $f(p, q)$ we set

(D.0)

$$
f(1,1)=2
$$

on $Z_{0} \times Z_{0}$,

(D.1) $\quad f\left(x^{i} r, x^{j} s\right)=b_{j} f\left(x^{i-j} r, s\right) \quad\left(i \geqq j, i, j \neq 0, r \in Y_{n}, s \in Y_{m}\right)$

on $X_{n+1} \times X_{m+1}$, similarly

(D.2) $f\left(y^{i} r, y^{j} s\right)=d_{j} f\left(y^{i-j} r, s\right)\left(i \geqq j, i, j \neq 0, r \in X_{n}, s \in X_{m}\right)$

on $Y_{n+1} \times Y_{m+1}$, while on $X_{n+1} \times Y_{m+1}$ we set

$$
f\left(x^{i}, y^{j}\right)=e_{i, j}
$$


(D.3)

$$
\begin{aligned}
& f\left(x^{i} r, y^{j} s\right)=e_{i, j} f(r, s)-f\left(y^{j} r, x^{i} s\right) \\
& \left(i, j \neq 0, r \in Y_{n}, s \in X_{m}, n+m>0\right)
\end{aligned}
$$

(where the latter term is already defined since $\left(y^{j} r, x^{i} s\right)$ has lower height if $n+m>0$-note $x^{i} s$ has the same height as $s$ if $s \in X_{m}$ for $m>0$, and similarly for $y^{j} r$ ), on $X_{n+1} \times Z_{0}$ we put

$$
\begin{array}{r}
f\left(x^{i}, 1\right)=a_{i} \\
f\left(x^{i} r, 1\right)=a_{i} f(r, 1)-f\left(r, x^{i}\right) \quad\left(i \neq 0, r \in Y_{n}, n>0\right)
\end{array}
$$

and similarly on $Y_{n+1} \times Z_{0}$

$$
f\left(y^{i}, 1\right)=c_{i}
$$

$$
f\left(y^{i} r, 1\right)=c_{i} f(r, 1)-f\left(r, y^{i}\right) \quad\left(i \neq 0, r \in X_{n}, n>0\right)
$$

(where in both cases the latter terms have been defined by (D.1)(D.3) if $n>0$ ).

Comparing (0D.0)-(0D.2) with (D.0)-(D.2) we see that at each step we have (6), and we easily verify that at each stage we also have (5) (so the $f(p), f(p, q)$ are indeed preimages of the $f_{s}(p), f_{s}(p, q)$ ). All that remains is to verify (07) and (7).

If we can establish

(i ) $b_{k} f\left(x^{i} r\right)=f\left(x^{k+i} r\right) \quad\left(k, i \neq 0, r \in Y_{n}\right)$

(ii) $e_{k, l} f\left(x^{i} r\right)=f\left(x^{k+i} r, y^{l} x^{i} r\right)$

$\left(i \neq 0\right.$ but $k=0, l=0$ allowed, $\left.r \in Y_{n}\right)$

(i) $b_{k} f\left(x^{i} r, x^{j} s\right)=f\left(x^{k+i} r, x^{k+j} s\right) \quad\left(i, j, k \neq 0, r \in Y_{n}, s \in Y_{m}\right)$

(ii) $b_{k} f\left(x^{i} r, y^{j} s\right)=f\left(x^{k+i} r, x^{k} y^{j} s\right) \quad\left(i, j, k \neq 0, r \in Y_{n}, s \in X_{m}\right)$

(iii) $b_{k} f\left(x^{i} r, 1\right)=f\left(x^{k+i} r, x^{k}\right) \quad\left(i, k \neq 0, r \in Y_{n}\right)$

(iv) $e_{k, l} f\left(x^{i} r, x^{j} s\right)=f\left(x^{k+i}, y^{l} x^{j} s\right)+f\left(y^{l} x^{i} r, x^{k+j} s\right)$

$\left(i, j \neq 0\right.$ but $k=0, l=0$ allowed, $\left.r \in Y_{n}, s \in Y_{m}\right)$

(v) $a_{k} f\left(x^{i} r, y^{j} s\right)=f\left(x^{k+i} r, y^{j} s\right)+f\left(x^{i} r, x^{k} y^{j} s\right)$

$\left(i, j, k \neq 0, r \in Y_{n}, s \in X_{m}\right)$

(vi) $\quad a_{k} f\left(x^{i} r, 1\right)=f\left(x^{k+i} r, 1\right)+f\left(x^{i} r, x^{k}\right)$

$\left(i, k \neq 0, r \in Y_{n}\right)$

then (07) and (7) will follow from the definitions, (08), (8), and their duals (replacing $x$ by $y, a$ by $c, b$ by $d$ ) according to the following table of possibilities:

\begin{tabular}{lccccc}
\multicolumn{1}{c}{$p$} & $07 . \mathrm{i}$ & $07 . \mathrm{iv}$ & $07 . \mathrm{ii}$ & $07 . \mathrm{v}$ & $07 . \mathrm{ii}$ \\
\hline$X_{n+1}$ & $08 . \mathrm{i}$ & $08 . \mathrm{ii}$ & $08 . \mathrm{ii}$ & $08 . \mathrm{ii}$ & $0 \mathrm{D} .2$ \\
$Z_{0}$ & $0 \mathrm{D} .1$ & $0 \mathrm{D} .4$ & $0 \mathrm{D} .3$ & $0 \mathrm{D} .5$ & $0 \mathrm{D} .2$ \\
$Y_{n+1}$ & $0 \mathrm{D} .1$ & $08 . \mathrm{ii}^{*}$ & $08 . \mathrm{ii}^{*}$ & $08 . \mathrm{ii}^{*}$ & $08 . \mathrm{i}^{*}$
\end{tabular}




\begin{tabular}{clllll}
$(p, q)$ & $7 . \mathrm{i}$ & $7 . \mathrm{iv}$ & $7 . \mathrm{iii}$ & $7 . \mathrm{v}$ & $7 . \mathrm{ii}$ \\
\hline$X_{n+1} \times X_{m+1}$ & $8 . \mathrm{i}$ & $8 . \mathrm{iv}$ & $8 . \mathrm{iv}$ & $8 . \mathrm{iv}$ & $\mathrm{D} .2$ \\
$X_{n+1} \times Z_{0}$ & $8 . \mathrm{iii}$ & $8 . \mathrm{vi}$ & $\mathrm{D} .3$ & $\mathrm{D} .5$ & $\mathrm{D} .2$ \\
$X_{n+1} \times Y_{m+1}$ & $8 . \mathrm{ii}$ & $8 . \mathrm{v}$ & $\mathrm{D} .3$ & $8 . \mathrm{v}^{*}$ & $8 . \mathrm{ii}^{*}$ \\
$Z_{0} \times Z_{0}$ & D.1 & $\mathrm{D} .4$ & $\mathrm{D} .3$ & $\mathrm{D} .5$ & $\mathrm{D} .2$ \\
$Y_{n+1} \times Z_{0}$ & D.1 & D.4 & D.3 & $8 . \mathrm{vi}^{*}$ & $8 . \mathrm{iii}^{*}$ \\
$Y_{n+1} \times Y_{m+1}$ & $\mathrm{D} .1$ & $8 . \mathrm{iv}^{*}$ & $8 . \mathrm{iv}^{*}$ & $8 . \mathrm{iv}^{*}$ & $8 . \mathrm{i}^{*}$
\end{tabular}

It remains to establish (08) and (8). We proceed by induction, assuming the results for all lesser heights (where we count $f(p)$ as having the same height as $f(p, p))$. (08.i) is quite trivial:

$$
\begin{aligned}
b_{k} f\left(x^{i} r\right) & =b_{k} b_{i} f(r) \\
& =b_{i+k} f(r) \\
& =f\left(x^{i+k} r\right)
\end{aligned}
$$

(08.ii) is also easy:

$$
\begin{aligned}
e_{k, l} f\left(x^{i} r\right) & =e_{k, l} b_{i} f(r)(0 \mathrm{D} .1) \\
& =e_{k+i, l} a_{i}-e_{k+2 i, l} f(r)(4 . \mathrm{iii}) \\
& =e_{k+i, l} f\left(x^{i} r, r\right)-f\left(x^{k+2 i} r, y^{l} r\right) \text { (induction 07.iv, iii) } \\
& =f\left(y^{l} x^{i} r, x^{k+i} r\right) \text { (induction 7.iii) }
\end{aligned}
$$

where the inductions are legitimate since $r$ has lesser height than $x^{i} r$ and $\left(x^{i} r, r\right)$ lesser height than $\left(x^{i} r, x^{i} r\right)$. The remaining formulas 8.i-vi follow exactly as in [5, pp. 321-324], deleting all factors 2 (our present $a_{k}, c_{k}, e_{k, l}, f(p, q)$ correspond to $2 a_{k}, 2 c_{k}, 2 e_{k, l}, 2 f(p, q)$ in that paper, but $b_{k}, d_{k}, f(p)$ correspond to $\left.b_{k}, d_{k}, f(p, p)\right)$. The case 8.v for $k>i$ was not done explicitly [5, p. 324], but the whole point of introducing (4.iv) is to reduce the case $k>i$ to the case $k<i$ [5, p. 323].

REMARK. The only difference between the linear and the quadratic cases is that in the latter we must work with the $f(p)$ 's as well as the $f(p, q)$ 's, whereas in the former we needed only the $f(p, q)$ 's since $f(p, p)=2 f(p)$.

\section{PART II. MACDONALD'S THEOREM WITH INVERSES.}

1. Forms of Macdonald's theorem with inverses. Recall [6] that two elements $x$ and $y$ in a Jordan algebra are inverses if $U_{x} y=x, U_{x} y^{2}=1$. This is symmetric in $x$ and $y$, and we write $y=x^{-1}$. Invertibility of $x$ is equivalent to invertibility of the 
operator $U_{x}$, and

$$
U_{x^{-1}}=U_{x}^{-1} \text {. }
$$

If $Y$ is a subset of $X$ we can form the free unital quadratic Jordan algebra on $X$ with inverses $Y$ as the algebra $\mathscr{F} \mathscr{Q} \mathscr{J}(X / Y)=$ $\mathscr{F} \mathscr{Q} \mathscr{J}\left(X \cup Y^{\prime}\right) / \Re$ where $y \rightarrow y^{\prime}$ is a bijection of $Y$ on a set $Y^{\prime}$ disjoint from $X$, and $\Re$ is the ideal generated by all $U_{y} y^{\prime}-y$, $U_{y}\left(y^{\prime}\right)^{2}-1$ for $y$ in $Y$. This has the universal property that any map $\varphi$ of $X$ into a Jordan algebra $\Im$ such that all the elements $y \in Y$ are mapped into invertible elements $\varphi(y) \in \mathfrak{F}$ factors uniquely through a homomorphism $\hat{\varphi}: \mathscr{F} \mathscr{Q} \mathscr{J}(X / Y) \rightarrow \mathfrak{F}$.

Just as $\mathscr{F} \mathscr{Q} \mathscr{J}(X) \subset \mathscr{F} \mathscr{Q} \mathscr{J}\left(X^{\prime}\right)$ if $X \subset X^{\prime}$, we have

$$
\mathscr{F} \mathscr{Q} \mathscr{J}(X / Y) \subset \mathscr{F} \mathscr{Q} \mathscr{J}\left(X^{\prime} / Y\right) \text { if } Y \subset X \subset X^{\prime} \text {. }
$$

(It is still an open question whether $\mathscr{F} \mathscr{Q} \mathscr{J}(X / Y) \subset \mathscr{F} \mathscr{Q} \mathscr{J}\left(X / Y^{\prime}\right)$ if $Y \subset Y^{\prime} \subset X[5$, p. 325]). We again have $\mathscr{F} \mathscr{Q} \mathscr{F}(X / Y) * \mathscr{F} \mathscr{Q} \mathscr{J}(Z)=$ $\mathscr{F} \mathscr{Q} \mathscr{J}(X \cup Z / Y)$ if $X$ and $Z$ are disjoint (as in (2)), so as a second corollary to Theorem 1 we have

THEOREm 3. The universal unital quadratic envelope for the free unital quadratic Jordan algebra $\mathfrak{J}^{(n-1 / m)}$ on $n-1$ generators and the inverses of the first $m$ generators is isomorphic to the multiplication algebra of $\mathfrak{\Im}^{(n-1 / m)}$ on $\mathfrak{\Im}^{(n / m)}$,

$$
\mathscr{U} \mathscr{Q} \mathscr{C}\left(\Im^{(n-1 / m)}\right)=\mathscr{C}\left(\mathfrak{\Im}^{(n-1 / m)} \mid \Im^{(n / m)}\right) .
$$

The free special Jordan algebra with inverses $\mathscr{F} \mathscr{S} \mathscr{J}(X / Y)$ is the (Jordan) subalgebra of the free associative algebra with inverses $\mathscr{F} \mathscr{A}(X / Y)$ generated by the elements $x \in X$ and $y^{-1}$ for $y \in Y$. Again we denote the free special Jordan (resp. free associative) algebra on $n$ generators $x_{1}, \cdots, x_{n}$ and the inverses $x_{1}^{-1}, \cdots, x_{m}^{-1}$ of the first $m$ generators by $\mathfrak{\Im}_{s}^{(n / m)}=\mathscr{F} \mathscr{S} \mathscr{J}\left(\left\{x_{1}, \cdots, x_{n}\right\} /\left\{x_{1}, \cdots, x_{m}\right\}\right)$ (resp. $\left.\mathfrak{U}^{(n / m)}=\mathscr{F} \mathscr{A}\left(\left\{x_{1}, \cdots, x_{n}\right\} /\left\{x_{1}, \cdots, x_{m}\right\}\right)\right)$. In this notation

$$
\mathfrak{\Im}_{s}^{(n-1 / m)} \subset \mathfrak{\Im}_{s}^{(n / m)} \subset \mathfrak{U}^{(n / m)+} .
$$

THEOREM 4. The universal unital compound linear envelope of the free special unital quadratic Jordan algebra $\mathfrak{\Im}_{s}^{(n-1 / m)}$ on $n-1$ generators and the inverses of the first $m$ is canonically isomorphic to the multiplication algebra of $\mathfrak{\Im}_{s}^{(n-1 / m)}$ on $\mathfrak{\Im}_{s}^{(n / m)}$,

$$
\mathscr{U} \mathscr{C} \mathscr{E}\left(\Im_{s}^{(n-1 / m)}\right)=\mathscr{L}\left(\mathfrak{\Im}_{s}^{(n-1 / m)} \mid \mathfrak{\Im}_{s}^{(n / m)}\right) .
$$

The proof is exactly as in the inverse-less case (again the uni- 
versal unital linear envelope of $\mathfrak{\Im}_{s}^{(n / m)}$ is $\left.\mathfrak{I}^{(n / m)}\right)$. From these two results we obtain as before

Equivalence Theorem with Inverses. The following conditions are equivalent for a given $m<n$ :

(I ) $\AA^{(n / m)} \cap 3^{(n / m)}=0$

(II) $\nu^{(n / m)}: 3^{(n / m)} \rightarrow 3_{s}^{(n / m)}$ is bijective

(III) $\mathscr{C l}\left(\mathcal{\nu}^{(n / m)}\right): \mathscr{l l}\left(\mathfrak{\Im}^{(n-1 / m)} \mid \mathfrak{\Im}^{(n / m)}\right) \rightarrow \mathscr{C l}\left(\mathfrak{\Im}_{s}^{(n-1 / m)} \mid \mathfrak{\Im}_{s}^{(n / m)}\right)$ is an isomorphism

(IV) the canonical homomorphism

$$
\mathscr{U} \mathscr{Q} \mathscr{E}\left(\mathfrak{\Im}^{(n-1 / m)}\right) \longrightarrow \mathscr{U} \mathscr{C} \mathscr{E}\left(\mathfrak{\Im}_{s}^{(n-1 / m)}\right)
$$

is an isomorphism

(V) all unital quadratic representations of $\mathfrak{\Im}^{(n-1 / m)}$ are special where $\mathfrak{\Im}^{(n / m)}, \Im_{s}^{(n / m)}$ denote the free and free special unital quadratic Jordan algebra on $n$ generators and the inverses of the first $m, \Omega^{(n / m)}$ denotes the kernel of the canonical homomorphism $\nu^{(n / m)}: \Im^{(n / m)} \rightarrow \Im_{s}^{(n / m)}$, and $\mathfrak{Z}^{(n / m)}, 3_{s}^{(n / m)}$ the subspaces of $\mathfrak{\Im}^{(n / m)}, \mathfrak{\Im}_{s}^{(n / m)}$ respectively consisting of elements linear in the variable $x_{n}$.

These conditions fail for $n \geqq 4$, but for $n=3$ we have

MACDONALD'S THEOREM WITH INVERSES. If an identity in the variables $x, x^{-1}, y, y^{-1}, z$ which is linear in $z$ holds for all unital special Jordan algebras, it holds for all unital quadratic Jordan algebras.

We now turn to the problem of finding generators and relations for $\mathscr{U} \mathscr{Q} \mathscr{C}\left(\mathfrak{\Im}^{(2 / 2}\right)$. The first result about generators is

Proposition 2. If $\Re$ is a unital quadratic Jordan algebra and $\Im$ a subalgebra generated by the elements of a unital subset. $X$ and the inverses of the elements $Y \subset X$ then the multiplication algebra $\mathscr{C l}(\Im) \Re)$ is generated by the transformations $U_{x}, U_{x, z}$ for $x, z$ in $X$ and the inverses $U_{y}^{-1}$ for $y$ in $Y$.

Proof. Since $\mathfrak{\Im}$ is generated by $X \cup Y^{-1}$ the general result [4, p. 000] says $\mathscr{C}(\mathfrak{\Im} \mid \Re)$ is generated by the $U_{z}, U_{z, w}$ for $z, w$ in $X \cup Y^{-1}$. That the $U_{y^{-1, z^{-1}}}$ and $U_{x, y^{-1}}$ are superfluous follows from the following relations:

Proposition 3. If $y$ and $z$ are invertible elements of a unital quadratic Jordan algebra then 


$$
\begin{gathered}
U_{y^{-1}}=U_{y}^{-1} \\
U_{y^{-1, x}}=U_{y}^{-1} V_{y, x}=V_{x, y} U_{y}^{-1} \\
V_{y^{-1}}=U_{y}^{-1} V_{y}=V_{y} U_{y}^{-1} \\
V_{y^{-1, x}}=U_{y}^{-1} U_{x, y}, \quad V_{x, y^{-1}}=U_{x, y} U_{y}^{-1} \\
U_{y^{-1, z}-^{-1}}=U_{y}^{-1} U_{y, z} U_{z}^{-1}=U_{z}^{-1} U_{y, z} U_{y}^{-1} .
\end{gathered}
$$

Proof. We have already seen (9). For (10) we cancel $U_{y}$ from the left and right of $U_{y} V_{x, y}=V_{y, x} U_{y}=U_{U(y) x, y}$ (by $U Q J$ III) $=$ $U_{U(y) x, U(y) y^{-1}}=U_{y} U_{x, y^{-1}} U_{y}$ (by UQJ II). We obtain (11) by setting $x=1$ in (10), and (12) by replacing $y$ by $y^{-1}$ in (10) (or by using (10), (11), and the definition of $V_{y^{-1, x}}$ ). From these we derive (13) by $U_{y^{-1, z^{-1}}}=U_{y}^{-1} V_{y, z^{-1}}$ (by (10)) $=U_{y}^{-1} U_{y, z} U_{z}^{-1}$ (by (12)).

Since $\mathscr{Q} \mathscr{Q} \mathscr{E}\left(\Im^{(2 / 2)}\right)$ has the form $\mathscr{C}\left(\Im^{(2 / 2)} \mid \Re\right)$ by Theorem 1 this suggests that we should represent $\mathscr{U} \mathscr{Q} \mathscr{E}\left(\mathfrak{\Im}^{(2 / 2)}\right)$ by

$$
\mathfrak{F}^{\prime} \longrightarrow \mathscr{U} \mathscr{Q} \mathscr{E}\left(\mathfrak{\Im}^{(2 / 2)}\right)
$$

where $\mathfrak{F}^{\prime}$ is the free associative algebra on the generators $a, b, c, d, e$ and the inverses $b^{-1}, d^{-1}$, and $a \rightarrow v(x), b \rightarrow u(x), c \rightarrow v(y), d \rightarrow u(y)$, $e \rightarrow u(x, y), b^{-1} \rightarrow u\left(x^{-1}\right), d^{-1} \rightarrow u\left(y^{-1}\right)$. The Proposition 2 guarantees that this map is surjective. We claim that its kernel $\mathscr{R}^{\prime}$ is generated by the same relations (R1)-(R6) as in the inverse-less case, together with the additional relations

(R7) $b b^{-1}=b^{-1} b=1, d d^{-1}=d^{-1} d=1$

(R8) $b^{-1} e d^{-1}=d^{-1} e b^{-1}$.

Presentation Theorem With Inverses. The universal unital quadratic envelope $\mathscr{Q} \mathscr{Q} \mathscr{E}\left(\mathfrak{\Im}^{(2 / 2)}\right)$ of the free unital quadratic Jordan algebra on two generators $x, y$ and their inverses $x^{-1}, y^{-1}$ is given abstractly by the eight generators $1, v(x), u(x), v(y), u(y), u(x, y), u\left(x^{-1}\right)$, $u\left(y^{-1}\right)$ and the eight pairs of relations

$$
\begin{array}{cc}
\text { ( i ) } & u(x) v(x)=v(x) u(x), \quad u(y) v(y)=v(y) u(y) \\
\text { ( ii ) } & u(x) v(y)+u(x, y) v(x)=v(y) u(x)+v(x) u(y, x), \\
& u(y) v(x)+u(y, x) v(y)=v(x) u(y)+v(y) u(x, y) \\
\text { (iii ) } & u(x) v(y, x)=v(x, y) u(x), \quad u(y) v(x, y)=v(y, x) u(y) \\
\text { (iv) } & u(x) v(y, y)+u(x, y) v(y, x)=v(y, y) u(x)+v(x, y) u(x, y), \\
& u(y) v(x, x)+u(y, x) v(x, y)=v(x, x) u(y)+v(y, x) u(y, x) \\
\text { ( v ) } & u(x) v\left(y^{2}, x\right)=v\left(x, y^{2}\right) u(x), \quad u(y) v\left(x^{2}, y\right)=v\left(y, x^{2}\right) u(y) \\
\text { ( vi ) } & u(x) v\left(y^{3}, x\right)=v\left(x, y^{3}\right) u(x), \quad u(y) v\left(x^{3}, y\right)=v\left(y, x^{3}\right) u(y) \\
\text { (vii) } & u(x) u\left(x^{-1}\right)=u\left(x^{-1}\right) u(x)=1, \quad u(y) u\left(y^{-1}\right)=u\left(y^{-1}\right) u(y)=1 \\
\text { (viii) } & u\left(x^{-1}\right) u(x, y) u\left(y^{-1}\right)=u\left(y^{-1}\right) u(x, y) u\left(x^{-1}\right) .
\end{array}
$$


At this point we merely want to prove that R1-R8 imply the analogues of R1-R6 where one or both of $x, y$ is replaced by $x^{-1}, y^{-1}$ :

(R1 ) $a b=b a, c d=d c$

(R1' ) $a^{\prime} b^{\prime}=b^{\prime} a^{\prime}, c^{\prime} d^{\prime}=d^{\prime} c^{\prime}$

(R2) $a e+c b=e a+b c, c e+a d=e c+d a$

(R2' ) $a^{\prime} e^{\prime}+c^{\prime} b^{\prime}=e^{\prime} a^{\prime}+b^{\prime} c^{\prime}, c^{\prime} e^{\prime}+a^{\prime} d^{\prime}=e^{\prime} c^{\prime}+d^{\prime} a^{\prime}$

(R2") $a^{\prime} e^{\prime \prime}+c b^{\prime}=e^{\prime \prime} a^{\prime}+b^{\prime} c, c^{\prime} e^{\prime \prime \prime}+a d^{\prime}=e^{\prime \prime \prime} c^{\prime}+d^{\prime} a$

(R2'") $a e^{\prime \prime \prime}+c^{\prime} b=e^{\prime \prime \prime} a+b c^{\prime}, c e^{\prime \prime}+a^{\prime} d=e^{\prime \prime} c+d a^{\prime}$

(R3 ) $b\{c a-e\}=\{a c-e\} b, d\{a c-e\}=\{c a-e\} d$

(R3' ) $b^{\prime}\left\{c^{\prime} a^{\prime}-e^{\prime}\right\}=\left\{a^{\prime} c^{\prime}-e^{\prime}\right\} b^{\prime}, d^{\prime}\left\{a^{\prime} c^{\prime}-e^{\prime}\right\}=\left\{c^{\prime} a^{\prime}-e^{\prime}\right\} d^{\prime}$

(R3" ) $b^{\prime}\left\{c a^{\prime}-e^{\prime \prime}\right\}=\left\{a^{\prime} c-e^{\prime \prime}\right\} b^{\prime}, d^{\prime}\left\{a c^{\prime}-e^{\prime \prime \prime}\right\}=\left\{c^{\prime} a-e^{\prime \prime \prime}\right\} d^{\prime}$

$\left(\mathrm{R} 3^{\prime \prime \prime}\right) \quad b\left\{c^{\prime} a-e^{\prime \prime \prime}\right\}=\left\{a c^{\prime}-e^{\prime \prime \prime}\right\} b, d\left\{a^{\prime} c-e^{\prime \prime}\right\}=\left\{c a^{\prime}-e^{\prime \prime}\right\} d$

(R4) $b\left\{c^{2}-2 d\right\}+e\{c a-e\}=\left\{c^{2}-2 d\right\} b+\{a c-e\} e$, $d\left\{a^{2}-2 b\right\}+e\{a c-e\}=\left\{a^{2}-2 b\right\} d+\{c a-e\} e$

(R4' ) $b^{\prime}\left\{c^{\prime 2}-2 d^{\prime}\right\}+e^{\prime}\left\{c^{\prime} a^{\prime}-e^{\prime}\right\}=\left\{c^{\prime 2}-2 d^{\prime}\right\} b^{\prime}+\left\{a^{\prime} c^{\prime}-e^{\prime}\right\} e^{\prime}$, $d^{\prime}\left\{a^{\prime 2}-2 b^{\prime}\right\}+e^{\prime}\left\{a^{\prime} c^{\prime}-e^{\prime}\right\}=\left\{a^{\prime 2}-2 b^{\prime}\right\} d^{\prime}+\left\{c^{\prime} a^{\prime}-e^{\prime}\right\} e^{\prime}$

$\left(\mathrm{R} 4^{\prime \prime}\right) \quad b^{\prime}\left\{c^{2}-2 d\right\}+e^{\prime \prime}\left\{c a^{\prime}-e^{\prime \prime}\right\}=\left\{c^{2}-2 d\right\} b^{\prime}+\left\{a^{\prime} c-e^{\prime \prime}\right\} e^{\prime \prime}$, $d^{\prime}\left\{a^{2}-2 b\right\}+e^{\prime \prime \prime}\left\{a c^{\prime}-e^{\prime \prime \prime}\right\}=\left\{a^{2}-2 b\right\} d^{\prime}+\left\{c^{\prime} a-e^{\prime \prime \prime}\right\} e^{\prime \prime \prime}$

$\left(\mathrm{R} 4^{\prime \prime \prime}\right) \quad b\left\{c^{\prime 2}-2 d^{\prime}\right\}+e^{\prime \prime \prime}\left\{c^{\prime} a-e^{\prime \prime \prime}\right\}=\left\{c^{2}-2 d^{\prime}\right\} b+\left\{a c^{\prime}-e^{\prime \prime \prime}\right\} e^{\prime \prime \prime}$, $d\left\{a^{\prime 2}-2 b^{\prime}\right\}+e^{\prime \prime}\left\{a^{\prime} c-e^{\prime \prime}\right\}=\left\{a^{\prime 2}-2 b^{\prime}\right\} d+\left\{c a^{\prime}-e^{\prime \prime}\right\} e^{\prime \prime}$

(R5 ) $b\left\{\left(c^{2}-2 d\right) a-(c e-d a)\right\}=\left\{a\left(c^{2}-2 d\right)-(e c-a d)\right\} b$, $d\left\{\left(a^{2}-2 b\right) c-(a e-b c)\right\}=\left\{c\left(a^{2}-2 b\right)-(e a-c b)\right\} d$

(R5' ) $b^{\prime}\left\{\left(c^{\prime 2}-2 d^{\prime}\right) a^{\prime}-\left(c^{\prime} e^{\prime}-d^{\prime} a^{\prime}\right)\right\}=\left\{a^{\prime}\left(c^{\prime 2}-2 d^{\prime}\right)-\left(e^{\prime} c^{\prime}-a^{\prime} d^{\prime}\right)\right\} b^{\prime}$, $d^{\prime}\left\{\left(a^{\prime 2}-2 b^{\prime}\right) c^{\prime}-\left(a^{\prime} e^{\prime}-b^{\prime} c^{\prime}\right)\right\}=\left\{c^{\prime}\left(a^{\prime 2}-2 b^{\prime}\right)-\left(e^{\prime} a^{\prime}-c^{\prime} b^{\prime}\right)\right\} d^{\prime}$

$\left(\mathrm{R}^{\prime \prime}\right) \quad b^{\prime}\left\{\left(c^{2}-2 d\right) a^{\prime}-\left(c e^{\prime \prime}-d a^{\prime}\right)\right\}=\left\{a^{\prime}\left(c^{2}-2 d\right)-\left(e^{\prime \prime} c-a^{\prime} d\right)\right\} b^{\prime}$, $d^{\prime}\left\{\left(a^{2}-2 b\right) c^{\prime}-\left(a e^{\prime \prime \prime}-b c^{\prime}\right)\right\}=\left\{c^{\prime}\left(a^{2}-2 b\right)-\left(e^{\prime \prime \prime} a-c^{\prime} b\right)\right\} d^{\prime}$

$\left(\mathrm{R}^{\prime \prime \prime}\right) \quad b\left\{\left(c^{\prime 2}-2 d^{\prime}\right) a-\left(c^{\prime} e^{\prime \prime \prime}-d^{\prime} a\right)\right\}=\left\{a\left(c^{2}-2 d^{\prime}\right)-\left(e^{\prime \prime \prime} c^{\prime}-a d^{\prime}\right)\right\} b$, $d\left\{\left(a^{\prime 2}-2 b^{\prime}\right) c-\left(a^{\prime} e^{\prime \prime}-b^{\prime} c\right)\right\}=\left\{c\left(a^{\prime 2}-2 b^{\prime}\right)-\left(e^{\prime \prime} a^{\prime}-c b^{\prime}\right)\right\} d$

(R6 ) $b\left\{\left(c^{3}-3 c d\right) a-c(c e-d a)+d e\right\}=\left\{a\left(c^{3}-3 d c\right)-(e c-a d) c+e d\right\} b$, $d\left\{\left(a^{3}-3 a b\right) c-a(a e-b c)+b e\right\}=\left\{c\left(a^{3}-3 b a\right)-(e a-c b) a+e b\right\} d$

$\left(\mathrm{R} 6^{\prime}\right) \quad b^{\prime}\left\{\left(c^{\prime 3}-3 c^{\prime} d^{\prime}\right) a^{\prime}-c^{\prime}\left(c^{\prime} e^{\prime}-d^{\prime} a^{\prime}\right)+d^{\prime} e^{\prime}\right\}=$ $\left\{a^{\prime}\left(c^{3}-3 d^{\prime} c^{\prime}\right)-\left(e^{\prime} c^{\prime}-a^{\prime} d^{\prime}\right) c^{\prime}+e^{\prime} d^{\prime}\right\} b^{\prime}$, $d^{\prime}\left\{\left(a^{\prime 3}-3 a^{\prime} b^{\prime}\right) c^{\prime}-a^{\prime}\left(a^{\prime} e^{\prime}-b^{\prime} c^{\prime}\right)+b^{\prime} e^{\prime}\right\}=$ $\left\{c^{\prime}\left(a^{\prime 3}-3 b^{\prime} a^{\prime}\right)-\left(e^{\prime} a^{\prime}-c^{\prime} b^{\prime}\right) a^{\prime}+e^{\prime} b^{\prime}\right\} d^{\prime}$

$\left(\mathrm{R} 6^{\prime \prime}\right) \quad b^{\prime}\left\{\left(c^{3}-3 c d\right) a^{\prime}-c\left(c e^{\prime \prime}-d a^{\prime}\right)+d e^{\prime \prime}\right\}=$ $\left\{a^{\prime}\left(c^{3}-3 d c\right)-\left(e^{\prime \prime} c-a^{\prime} d\right) c+e^{\prime \prime} d\right\} b^{\prime}$, $d^{\prime}\left\{\left(a^{3}-3 a b\right) c^{\prime}-a\left(a e^{\prime \prime \prime}-b c^{\prime}\right)+b e^{\prime \prime \prime}\right\}=$ $\left\{c^{\prime}\left(a^{3}-3 b a\right)-\left(e^{\prime \prime \prime} a-c^{\prime} b\right) a+e^{\prime \prime \prime} b\right\} d^{\prime}$

$\left(\mathrm{R} 6^{\prime \prime \prime}\right) \quad b\left\{\left(c^{\prime 3}-3 c^{\prime} d^{\prime}\right) a-c^{\prime}\left(c^{\prime} e^{\prime \prime \prime}-d^{\prime} a\right)+d^{\prime} e^{\prime \prime \prime}\right\}=$ $\left\{a\left(c^{\prime 3}-3 d^{\prime} c^{\prime}\right)-\left(e^{\prime \prime \prime} c^{\prime}-a d^{\prime}\right) c^{\prime}+e^{\prime \prime \prime} d^{\prime}\right\} b$, $d\left\{\left(a^{\prime 3}-3 a^{\prime} b^{\prime}\right) c-a^{\prime}\left(a^{\prime} e^{\prime \prime}-b^{\prime} c\right)+\left(b^{\prime} e^{\prime \prime}\right\}=\right.$ $\left\{c\left(a^{\prime 3}-3 b^{\prime} a^{\prime}\right)-\left(e^{\prime \prime} a^{\prime}-c b^{\prime}\right) a^{\prime}+e^{\prime \prime} b^{\prime}\right\} d$

where (as in (9)-(13)) we set 


$$
\begin{gathered}
a^{\prime}=b^{-1} a=a b^{-1}, c^{\prime}=d^{-1} c=c d^{-1}, b^{\prime}=b^{-1}, d^{\prime}=d^{-1}, \\
e^{\prime \prime}=b^{-1}\{a c-e\}=\{c a-e\} b^{-1}, e^{\prime \prime \prime}=d^{-1}\{c a-e\}=\{a c-e\} d^{-1}, \\
e^{\prime}=b^{-1} e d^{-1}=d^{-1} e b^{-1} .
\end{gathered}
$$

(These definitions are consistent by R1, R7; by R3; and by R8). Note this implies

$$
\begin{gathered}
b a^{\prime}=a^{\prime} b=a, d c^{\prime}=c^{\prime} d=c \\
e^{\prime \prime} d^{\prime}=a^{\prime} c^{\prime}-e^{\prime}, d^{\prime} e^{\prime \prime}=c^{\prime} a^{\prime}-e^{\prime} \\
e^{\prime \prime \prime} b^{\prime}=c^{\prime} a^{\prime}-e^{\prime}, b^{\prime} e^{\prime \prime \prime}=a^{\prime} c^{\prime}-e^{\prime} \\
b e^{\prime} d=d e^{\prime} b=e .
\end{gathered}
$$

Since $a^{\prime}, b^{\prime}, c^{\prime}, d^{\prime}, e^{\prime}, e^{\prime \prime}, e^{\prime \prime \prime}$ are symmetric under the reversal involution in $\mathfrak{F}$, these formulas merely amount to saying that the left sides are symmetric under reversal. Thus in establishing them we consider only the left sides, and show that in each case these are indeed symmetric. Furthermore, by symmetry in $x$ and $y$ it is enough to check the first of each pair of relations. Finally, an element $S$ will be symmetric if $b S b$ (or $b d S d b$ ) is, so it suffices if $S$ becomes symmetric upon multiplication on the right and left by $b$ (or on the right by $d b$ and on the left by $b d$ ).

The relations R1-R6 are hypotheses, and the relation $\mathrm{R} 1$ ' follows from R1 via R7.

The left side of the first of each pair of relations $R 2^{\prime}-\mathrm{R} 6^{\prime}$ becomes symmetric upon multiplying on the left by $d b$ and on the right by bd. Thus for R2'

$$
\begin{aligned}
d b\left\{a^{\prime} e^{\prime}+c^{\prime} b^{\prime}\right\} b d=d a d^{\prime} e d+d b c \\
\quad=d a d^{\prime}(e-c a) d+d a d^{\prime} c a d+d b c \\
=d a(e-a c)+d b c+d a\left(d^{\prime} c\right) a d(\mathrm{by} \text { R3) } \\
\quad=d\left\{a e-a^{2} c+b c\right\}+d a\left(d^{\prime} c\right) a d
\end{aligned}
$$

which is symmetric by $\mathrm{R} 1$ and R5. For R3',

$$
d b\left\{b^{\prime}\left(c^{\prime} a^{\prime}-e^{\prime}\right)\right\} b d=(c a-e) d
$$

is symmetric by R3. R4' is a little harder; we will find it convenient to drop out symmetric terms as they appear rather than carry them along at each stage. We have

$$
\begin{aligned}
& d b\left\{b^{\prime}\left(c^{\prime 2}-2 d^{\prime}\right)+e^{\prime}\left(c^{\prime} a^{\prime}-e^{\prime}\right)\right\} b d \\
& =c^{\prime} c b d-2 b d+d e d^{\prime 2} c a d-d b e^{2} b d \\
& \cong c^{\prime}\left\{-\left[e a-c a^{2}\right] d+d\left[(a e-b c)-\left(a^{2}-2 b\right) c\right]\right\}-2 b d \\
& +\{d a c+e d-c a d\} d^{\prime 2} c a d \text { (by R5.R3) }
\end{aligned}
$$




$$
\begin{aligned}
= & \left\{c d^{\prime}(c a-e) a d+c a e-c b c-c\left(a^{2}-2 b\right) c\right\}-2 b d \\
& +d a c d^{\prime 2} c a d+e c d^{\prime} a d-c a c d^{\prime} a d \\
\cong & c(a c-e) d^{\prime} a d+c a e-2 b d+e c d^{\prime} a d-c a c d^{\prime} a d(\mathrm{by} \mathrm{R} 3) \\
= & c a e-2 b d+(e c-c e) d^{\prime} a d \\
= & c a e-2 b d+(a d-d a) d^{\prime} a d(\mathrm{by} \mathrm{R} 2) \\
= & c a e-2 b d+a^{2} d-d a d^{\prime} a d \\
\cong & \left(a^{2}-2 b\right) d+c a e
\end{aligned}
$$

which is symmetric by $\mathrm{R} 4$. For R5',

$$
\begin{aligned}
d b b^{\prime} & \left\{\left(c^{\prime 2}-2 d^{\prime}\right) a^{\prime}-\left(c^{\prime} e^{\prime}-d^{\prime} a^{\prime}\right)\right\} b d \\
& =c c^{\prime} a d-2 a d-c d^{\prime} e d+a d \\
& =c d^{\prime}(c a-e) d-a d \\
& =c(a c-e)-a d(\mathrm{by} \mathrm{R} 3) \\
& =c a c-c e-a d
\end{aligned}
$$

which is symmetric by R2. Finally, for $R 6^{\prime}$

$$
\begin{aligned}
d b b^{\prime} & \left\{\left(c^{\prime 3}-3 c^{\prime} d^{\prime}\right) a^{\prime}-c^{\prime}\left(c^{\prime} e^{\prime}-d^{\prime} a^{\prime}\right)+d^{\prime} e^{\prime}\right\} b d \\
& =\left(c^{3} d^{\prime 2}-3 c d^{\prime}\right) a d-c\left(c d^{\prime 2} e d-d^{\prime} a d\right)+d^{\prime} e d \\
& =c^{2} d^{\prime 2}(c a-e) d-c d^{\prime} a d+d^{\prime}(e-c a) d \\
& =c^{2} d^{\prime}(a c-e)-c d^{\prime} a d+(e-a c)(\mathrm{by} \mathrm{R} 3) \\
& =c\left\{d^{\prime}(c a-e)\right\} c+c d^{\prime}(e c-c e-a d)+e-a c \\
& =c\left\{d^{\prime}(c a-e)\right\} c-c d^{\prime}(d a)-a c+e(\mathrm{by} \mathrm{R} 2) \\
& =c\left\{d^{\prime}(c a-e)\right\} c-(c a+a c)+e
\end{aligned}
$$

is symmetric by R3.

The first of each pair of relations $R 2^{\prime \prime}-\mathrm{R} 6^{\prime \prime}$ becomes symmetric by multiplying on the left and right by $b$. Thus for R2"

$$
b\left\{a^{\prime} e^{\prime \prime}+c b^{\prime}\right\} b=a(c a-e)+b c=a c a-a e+b c
$$

is symmetric by $\mathrm{R} 2$, for $\mathrm{R} 3{ }^{\prime \prime}$

$$
b\left\{b^{\prime}\left(c a^{\prime}-e^{\prime \prime}\right)\right\} b=c a-(c a-e)=e
$$

is clearly symmetric, for $\mathrm{R} 4^{\prime \prime}$

$$
\begin{aligned}
& b\left\{b^{\prime}\left(c^{2}-2 d\right)+e^{\prime \prime}\left(c a^{\prime}-e^{\prime \prime}\right)\right\} b \\
& \quad=\left(c^{2}-2 d\right) b+(a c-e) c a-b e^{\prime \prime 2} b \\
& \quad=\left(c^{2}-2 d\right) b-e c a+a c^{2} a-b e^{\prime 2} b
\end{aligned}
$$

is symmetric by $R 4$, for $R 5^{\prime \prime}$

$$
\begin{aligned}
& b b^{\prime}\left\{\left(c^{2}-2 d\right) a^{\prime}-\left(c e^{\prime \prime}-d a^{\prime}\right)\right\} b \\
& \quad=\left(c^{2}-2 d\right) a-c(c a-e)+d a
\end{aligned}
$$




$$
=-d a+c e
$$

is symmetric by $\mathrm{R} 2$, and for $\mathrm{R} 6^{\prime \prime}$

$$
\begin{aligned}
b b^{\prime}\left\{\left(c^{3}-3 c d\right) a^{\prime}-c\left(c e^{\prime \prime}-d a^{\prime}\right)+d e^{\prime \prime}\right\} b \\
\quad=\left(c^{3}-3 c d\right) a-c^{2}(c a-e)+c d a+d(c a-e) \\
\quad=-c d a+c^{2} e-d e \\
\quad=c\{c e-d a\}-d e \\
\quad=c\{e c-a d\}-d e(\text { by R2) } \\
\quad=c e c-c a d-d e
\end{aligned}
$$

is symmetric by $\mathrm{R} 3$.

Exactly the same arguments (replacing $a, a^{\prime}, b, b^{\prime}, c, d, e, e^{\prime \prime}$ by $a^{\prime}, a, b^{\prime}, b, c^{\prime}, d^{\prime}, e^{\prime}, e^{\prime \prime \prime}$ and using R2', R4', R3'-which we have already established-instead of R2, R4, R3) establish the symmetry of the left sides of the first of each pair of relations $\mathrm{R} 2^{\prime \prime \prime}-\mathrm{R} 6^{\prime \prime \prime}$.

2. The crucial lemma again. Once again the key to the theorems is the existence of certain elements in the algebra $\mathfrak{F}^{\prime} / \mathbb{R}^{\prime}$.

Lemma 2. In the algebra $\mathfrak{F}^{\prime} / \mathbb{R}^{\prime}$ there are elements $a_{k}, b_{k}, c_{k}, d_{k}, e_{k, l}$ (for all integers $k, l$ ) satisfying

$$
\begin{array}{ll}
\text { (i) } \quad & a_{0}=c_{0}=e_{0,0}=2, b_{0}=d_{0}=1 \\
& a_{1}=e_{1,0}=a, a_{-1}=e_{-1,0}=b^{-1} a=a b^{-1}, b_{1}=b, b_{-1}=b^{-1} \\
& c_{1}=e_{0,1}=c, c_{-1}=e_{0,-1}=d^{-1} c=c d^{-1}, d_{1}=d, d_{-1}=d^{-1} \\
& e_{1,1}=e, e_{-1,-1}=b^{-1} e d^{-1}=d^{-1} e b^{-1} \\
& e_{1,-1}=d^{-1}\{c a-e\}=\{a c-e\} d^{-1}, e_{-1,1}=b^{-1}\{a c-e\}=\{c a-e\} b^{-1} \\
\text { (ii) } & b_{i} b_{j}=b_{i+j}, d_{i} d_{j}=d_{i+j} \\
& {\left[a_{i}, b_{j}\right]=\left[a_{i}, a_{j}\right]=0,\left[c_{i}, d_{j}\right]=\left[c_{i}, c_{j}\right]=0} \\
\text { (iii) } e_{i+k, j}=a_{k} e_{i, j}-b_{k} e_{i-k, j}=e_{i, j} a_{k}-e_{i-k, j} b_{k} \\
& e_{j, i+k}=c_{k} e_{j, i}-d_{k} e_{j, i-k}=e_{j, i} c_{k}-e_{j, i-k} d_{k} \\
\text { (iv) } & e_{-i,-j}=b_{-i} e_{i, j} d_{-j}=d_{-j} e_{i, j} b_{-i} \\
& e_{-i, j}=b_{-i} f_{i, j}=g_{i, j} b_{-i} \quad\left(f_{i, j}=a_{i} c_{j}-e_{i, j}\right) \\
& e_{i,-j}=d_{-j} g_{i, j}=f_{i, j} d_{-j} \quad\left(g_{i, j}=c_{j} a_{i}-e_{i, j}\right) .
\end{array}
$$

Proof. Again the first step is to construct the $e_{i, j}$. For $i, j \geqq 0$ this goes exactly as in the inverse-less case. We can also copy this procedure for $i, j \leqq 0$ or $j \geqq 0 \geqq i$ or $i \geqq 0 \geqq j$ since the $a^{\prime}=b^{-1} a=$ $a b^{-1}, b^{\prime}=b^{-1}, c^{\prime}=d^{-1} c=c d^{-1}, d^{\prime}=d^{-1}, e^{\prime}=b^{-1} e d^{-1}=d^{-1} e b^{-1}$ or $a^{\prime}, b^{\prime}, c, d, e^{\prime \prime \prime}=$ $b^{-1}(a c-e)=(c a-e) b^{-1}$ or $a, b, c^{\prime}, d^{\prime}, e^{\prime \prime \prime}=d^{-1}(c a-e)=(a c-e) d^{-1}$ satisfy the same axioms as $a, b, c, d, e$ by $\mathrm{R} 1^{\prime}-\mathrm{R} 6^{\prime}$ or $\mathrm{R} 1^{\prime \prime}-\mathrm{R} 6^{\prime \prime}$ or $\mathrm{R} 1^{\prime \prime \prime}-\mathrm{R} 6^{\prime \prime}$.

Given the $e_{i, j}$ we define $a_{i}=e_{i, 0}, b_{i}=b^{i}, c_{i}=e_{0, i}, d_{i}=d^{i}$ for all 
(positive or negative) $i$. From these (i) and (ii) follow immediately (using the commutativity R1).

Our next step is to get the intrinsic formulas (iv) for the $e_{i, j}$ involving negative indices rather than merely recursion formulas. It will suffice to prove the second relation, for the third follows similarly, and the second and third together imply the first. (Note that because $a_{i}=e_{i, 0}, c_{i}=e_{0, i}$ we have

$$
f_{i+2, j}=a f_{i+1, j}-b f_{i, j}, g_{i+2, j}=g_{i+1, j} a-g_{i, j} b
$$

for $i \geqq 0$, and similarly for $f_{-i-2, j}$ and $g_{-i-2, j}$ using $a^{\prime}, b^{\prime}$ in place of $a, b$, while

$$
f_{i, j+2}=f_{i, j+1} c-f_{i, j} d, g_{i, j+2}=c g_{i, j+1}-d g_{i, j}
$$

for $j \geqq 0$, and similarly for $f_{i,-j-2}$ and $g_{i,-j-2}$ using $c^{\prime}, d^{\prime}$ in place of $c, d$. These are similar to the relations defining the $e_{i, j}$, except for a lack of symmetry). We induct on $|i|+|j|$. The result is true by definition if $0 \leqq|i|,|j| \leqq 1$, and using induction

$$
\begin{aligned}
e_{i+2,-j} & =a e_{i+1,-j}-b e_{i,-j} \text { (definition) } \\
& =\left\{a f_{i+1, j}-b f_{i, j}\right\} d_{-j} \text { (induction) } \\
& =f_{i+2, j} d_{-j}
\end{aligned}
$$

if $i \geqq 0$, similarly for $e_{i-2,-j}$ if $i \leqq 0$ using $a^{\prime}, b^{\prime}$ in place of $a, b$, while if $j \geqq 0$

$$
\begin{aligned}
e_{i,-j-2} & =e_{i,-j-1} c^{\prime}-e_{i,-j} d^{\prime} \text { (definition) } \\
& =f_{i, j+1} d_{-j-1} c^{\prime}-f_{i, j} d_{-j} d^{\prime} \text { (induction) } \\
& =\left\{f_{i, j+1} c-\cdot f_{i, j} d\right\} d_{-j-2} \text { (commutativity) } \\
& =f_{i, j+2} d_{-j-2}
\end{aligned}
$$

and similarly for $e_{i,-j+2}$ if $j \leqq 0$ using $c, d$ in place of $c^{\prime}, d^{\prime}$. This completes the induction on $i$ and $j$.

Now we turn to (iii). By symmetry it suffices to prove only the first of the four equalities. We know

$$
a_{k} e_{i, j}=e_{i+k, j}+b_{k} e_{i-k, j}
$$

if $i, j, k \geqq 0$ and $i \geqq k$ by the inverse-less case. If $i<k$, say $k=i+l$ for $l>0$, then

$$
\begin{gathered}
a_{i+l} e_{i, j}=e_{2 i+l, j}+b_{i} a_{l} c_{j}-b_{i} e_{l, j}(4 . \mathrm{iv}, \text { iii }) \\
=e_{2 i+l, j}+b_{i} b_{l} b_{-l} f_{l, j}\left(\text { by }\left(4^{\prime} . \mathrm{ii}\right)\right) \\
=e_{2 i+l, j}+b_{i+l} e_{-l, j}\left(\text { by }\left(4^{\prime} . \mathrm{iv}\right)\right) .
\end{gathered}
$$

This establishes the result for $i, j, k \geqq 0$. Since $a_{i}=e_{i, 0}$ it also implies

$$
a_{k} f_{i, j}=f_{i+k, j}+b_{k} f_{i-k, j} \text {. }
$$


The relations (iv) allow us to convert any $e_{i,-j}$ into an $f_{i, j}$ by multiplying on the right by $d_{j}$, similarly to convert an $e_{-i, j}$ or $f_{-i, j}$ into an $f_{i, j}$ or $e_{i, j}$ by multilplying on the left by $b_{i}$, and an $a_{-k}$ or $b_{-k}$ or 1 into an $a_{k}$ or 1 or $b_{k}$ by multiplying on the left by $b_{k}$, so we can reduce the general case to the case $i, j, k \geqq 0$.

3. Proof of the theorems. We again have surjections

$$
\mathfrak{F}^{\prime} / \mathfrak{R}^{\prime} \stackrel{\sigma^{\prime}}{\longrightarrow} \mathscr{U} \mathscr{Q} \mathscr{E}\left(\mathfrak{\Im}^{(2 / 2)}\right) \stackrel{\nu^{\prime}}{\longrightarrow} \mathscr{Q} \mathscr{C} \mathscr{E}\left(\mathfrak{\Im}^{(2 / 2)}\right) \stackrel{\tau^{\prime}}{\longrightarrow} 3_{s}^{(3 / 2)}
$$

and the theorems result by showing $\mu^{\prime}=\tau^{\prime} \circ \nu^{\prime} \circ \sigma^{\prime}$ is injective. We follow the same procedure as in the inverse-less case of finding inverse images of the basis $f_{s}(p), f_{s}(p, q)$ of $3_{s}^{(3 / 2)}$ where $p, q$ are now monomials in $\mathfrak{Y}^{(2 / 2)}$. We define the inverse images $f(p), f(p, q)$ recursively by (0D.0-0D.5), (D.0-D.5) as before (but now the exponents $i, j$ may be positive or negative). The derivation of (6), (07), (7) from (08) and (8) follows from the charts as before. The proof of (08) carries over verbatim to the case of inverses since the relations (4.i-iii) for positive $i, j, k$ carry over to the relations (4'.i-iii) for positive and negative $i, j, k$. The proof of (8) is again just a repeat of that given in [5, p. 321-324] (deleting all factors 2).

\section{PART III. SHIRSHOV'S THEOREM.}

Shirshov's Theorem says that the free unital quadratic Jordan algebra $\mathfrak{\Im}^{(2)}$ on two generators is special: the canonical homomorphism $\nu^{(2)}: \mathfrak{F}^{(2)} \rightarrow \mathfrak{F}_{s}^{(2)}$ is an isomorphism. In the case of linear algebras this follows immediately from Macdonald's Theorem since if $\nu^{(2)}(p)=0$ for some polynomial $p(x, y) \in \mathfrak{\Im}^{(2)}$ then any multiplication in $\mathscr{U} \mathscr{C} \mathscr{E}\left(\mathfrak{\Im}_{s}^{(2)}\right)$ involving $\nu^{(2)}(p)$ and (via the isomorphism $\mathscr{U} \mathscr{Q} \mathscr{E}\left(\Im^{(2)}\right) \rightarrow \mathscr{C} \mathscr{C} \mathscr{E}\left(\Im_{s}^{(2)}\right)$ ) any multiplication in $\mathscr{U} \mathscr{Q} \mathscr{E}\left(\mathfrak{\Im}^{(2)}\right)$ involving $p$ will be zero, and in a unital linear algebra $L_{p}=0$ implies $p=0$. In the quadratic case we have no operator $L_{p}$, but we can conclude $U_{p}=V_{p}=U_{p, q}=$ for any $q$; thus $p=0$ if $\mathfrak{\Im}^{(2)}$ has no extreme radical [4, p. 000], for example if $\frac{1}{2} \in \Phi$. In general it is an open question whether free algebras $\mathscr{F} \mathscr{L}(X)$ have zero extreme radical for arbitrary $\Phi$ (it is conjectured that they in fact have no zero divisors, and even are imbeddable in division algebras). Therefore there seems to be no way at present of obtaining Shirshov's Theorem directly from Macdonald's Theorem.

We know [4, p. 000] that $\mathfrak{\Im}^{(2)}$, being generated by $x$ and $y$, equals $\mathscr{L} 1+\mathscr{C} x+\mathscr{C} y$ for $\mathscr{C}=\mathscr{C}\left(\mathfrak{F}^{(2)}\right)$ the multiplication algebra of $\mathfrak{\Im}^{(2)}$. If $\hat{U}$ is the natural surjection $\mathscr{U} \mathscr{Q} \mathscr{E}\left(\mathfrak{\Im}^{(2)}\right) \rightarrow \mathscr{M}\left(\mathfrak{\Im}^{(2)}\right)$ extending the regular representation $U$ of $\mathfrak{\Im}^{(2)}$ in $\mathscr{M}\left(\mathfrak{\Im}^{(2)}\right)$ then the $m(p)=\hat{U}(f(p))$ 
and $m(p, q)=\hat{U}(f(p, q))$ span $\mathscr{C}\left(\mathfrak{\Im}^{(2)}\right)$ since the $f(p), f(p, q)$ span $\mathscr{U} \mathscr{Q} \mathscr{E}\left(\mathfrak{\Im}^{(2)}\right)$ by our proof of Macdonald's Theorem. Thus $\Im^{(2)}$ is spanned by the

$$
\begin{array}{rrr}
m(p) 1, & m(p) x, & m(p) y \\
m(p, q) 1, & m(p, q) x, & m(p, q) y
\end{array}
$$

From our definitions D.0-D.5 of the $f(p, q)$ we see that they all are linear combinations of terms of the form $g e_{i, j}$ where $i, j \geqq 0$ and $g$ is a monomial in the $a_{k}, b_{k}, c_{k}, d_{k}, e_{k, l}$. Furthermore, the formulas (4) for the $e_{i, j}$ show in turn that they are all linear combinations of terms of the form $g e_{0,0}, g e_{1,0}, g e_{0,1}$, or $g e_{1,1}$. Thus every $m(p, q)=\hat{U}(f(p, q))$ is a linear combination of terms of the form $m(2 I), m V_{x}, m V_{y}, m U_{x, y}$ for $m$ an $m(r)$ or $m(r, s)$ and $2 I=\hat{U}\left(e_{0,0}\right), V_{x}=\hat{U}(a)=\hat{U}\left(e_{1,0}\right), V_{y}=\hat{U}(c)=$ $\hat{U}\left(e_{0,1}\right), U_{x, y}=\hat{U}(e)=\hat{U}\left(e_{1,1}\right)$. But the action of these latter operators on $x$ can be expressed in terms of other operators acting on 1: $2 x=$ $V_{x} 1, V_{x} x=2 x^{2}=V_{x^{2}} 1, V_{y} x=y \circ x=U_{x, y} 1, U_{x, y} x=\{x x y\}=x^{2} \circ y=U_{x^{2}, y} 1$. This implies that any $m(p, q) x$ is a sum of terms of the form $m 1$ ( $m$ an $m(r)$ or $m(r, s)$ ); a similar result applies to the $m(p, q) y$, so that $\mathfrak{\Im}^{(2)}$ is spanned by all $m(p) 1, m(p) x, m(p) y, m(p, q) 1(p \neq q$ monomials in $\left.\mathfrak{A}^{(2)}\right)$. A similar result holds in $\mathfrak{\Im}_{s}^{(2)}$ : indeed, a basis for $\mathfrak{F}_{s}^{(2)}$ consists of all

$$
m_{s}(p) 1=p p^{*}, m_{s}(p) x=p x p^{*}, m_{s}(p) y=p y p^{*}, m_{s}(q, 1) 1=q+q^{*}
$$

for $p, q$ monomials in $\mathfrak{2}^{(2)}$ with $q^{*} \neq q$. It is easy to verify that the canonical homomorphism $\nu^{(2)}: \mathfrak{\Im}^{(2)} \rightarrow \mathfrak{J}_{s}^{(2)}$ sends the $m(p) 1, m(p) x, m(p) y$, $m(p, q) 1$ into $m_{s}(p) 1, m_{s}(p) x, m_{s}(p) y, m_{s}(p, q) 1$ respectively by our recursive definition of $f(p), f(p, q)$. If we can show that the

$$
m(p) 1, m(p) x, m(p) y, m(q, 1) 1 \quad\left(q \neq q^{*}\right)
$$

span $\Im^{(2)}$ then $\nu^{(?)}$ will take a spanning set onto a basis, hence a basis onto a basis, and thus will be a bijection. This will prove Shirshov's Theorem. Thus our only problem is to convert $m(p, q) 1$ 's into $m(r, 1) 1$ 's and $m(p, 1) 1$ 's for symmetric $p$ into $m(r) 1, m(r) x, m(r) y$ 's.

LEMMA 3. If $p, q$ are monomials in the free associative algebra $\mathfrak{A}^{(2)}$ and $m(p, q)=\hat{U}(f(p, q)) \in \mathscr{C}\left(\mathfrak{F}^{(2)}\right)$ then in $\mathfrak{\Im}^{(2)}$

$$
m(p, q) 1=m\left(p q^{*}, 1\right) 1
$$

and if $p$ is symmetric, $p=r z r *$ for $z=1, x$, or $y$, then

$$
m(p, 1) 1=2 m(r) z \text {. }
$$

Proof. Note that 


$$
m\left(x^{i} y^{j}, 1\right)=V_{x^{i}, y^{j}}, \quad m\left(x^{i}, y^{j}\right)=U_{x^{i}, y^{j}}, \quad m\left(x^{i}, 1\right)=V_{x^{i}}
$$

and in any Jordan algebra

$$
Z_{z, w} 1=U_{z, w} 1=z \circ w .
$$

Also recall that

$$
x^{i} \circ x^{j}=2 x^{i+j}
$$

(this is trivial for $i, j \leqq 1$, and if $j \geqq 2$ then $x^{i} \circ x^{j}=V_{x^{i}} U_{x} x^{j-2}=$ $U_{x} V_{x^{i}} x^{j-2}=2 U_{x} x^{i+j-2}=2 x^{i+j}$ by induction). Finally, observe that the $m$ 's multiply according to

$$
\begin{aligned}
& m(p, q) m(r, s)=m(p r, q s)+m(p s, q r) \\
& m(p) m(r, s)=m(p r, p s) \\
& m(r, s) m(p)=m(r p, s p)
\end{aligned}
$$

because this holds for the $f$ 's.

The second result follows directly from the first: $m(p, 1) 1=$ $m\left(r z r^{*}, 1\right) 1=m(r z, r) 1$ (by the first) $=m(r) m(z, 1) 1=2 m(r) z$.

For the first result we induct on the height $h(p)+h(q)$. The result is trivial if $q=1$, so we always assume $h(q) \geqq 1$. First assume $h(p) \geqq 3$, say $p=x^{i} y^{j} x^{k} r$ for $i, j, k>0$. Then

$$
\begin{aligned}
&\left\{m(p, q)-m\left(p q^{*}, 1\right)\right\} 1=\left\{m\left(x^{i} y^{j} x^{k} r, q\right)-m\left(x^{i} y^{j} x^{k} r q^{*}, 1\right)\right\} 1 \\
&= m\left(x^{i}, 1\right)\left\{m\left(y^{j} x^{k} r, q\right)-m\left(y^{j} x^{k} r q^{*}, 1\right)\right\} 1 \\
&-m\left(x^{i}, y^{j}\right)\left\{m\left(x^{k} r, q\right)-m\left(x^{k} r q^{*}, 1\right)\right\} 1 \\
&+\left\{m\left(x^{i+k} r, y^{j} q\right)-m\left(x^{i+k} r q^{*}, y^{j}\right)\right\} 1
\end{aligned}
$$

where by induction the three terms in braces kill 1 since they all have height less than $h(p)+h(q)$.

Now assume $h(p)=2$. If $h(q) \geqq 3$ write $q=r s$ for $h(r) \geqq 2$, $h(s) \geqq 1$ and $p=t w$ for $h(t)=h(w)=1$. Then

$$
\begin{aligned}
m(p, q) & 1=m(q, p) 1=m(r s, t w) 1 \\
= & m\left(r s w^{*} t^{*}, 1\right) 1((\mathrm{~A}): \text { since } h(r s) \geqq 3) \\
= & m\left(r s w^{*}, t\right) 1\left((\mathrm{~B}): \text { since } h\left(r s w^{*}\right) \geqq 3\right) \\
= & \left\{m(r, 1) m\left(t, s w^{*}\right)-m\left(r t, s w^{*}\right)\right\} 1 \\
= & \left\{m(r, 1) m\left(t w s^{*}, 1\right)-m\left(r t, s w^{*}\right)\right\} 1 \text { (by induction since } \\
& \left.h\left(t w s^{*}\right)<h(p)+h(q) \text { if } r \neq 1\right) \\
= & \left\{m\left(r, t w s^{*}\right)+m\left(r t w s^{*}, 1\right)-m\left(r t w s^{*}, 1\right)\right\} 1((\mathrm{C}): \text { since } \\
& h(r t) \geqq 2+1=3) \\
= & m\left(t w s^{*}, r\right) 1 \\
= & m\left(t w s^{*} r^{*}, 1\right) 1\left((\mathrm{D}): \text { since } h\left(t w s^{*}\right) \geqq 1+1+1=3\right) \\
= & m\left(p q^{*}, 1\right) 1 .
\end{aligned}
$$


The remaining cases $h(p)=2, h(q)=1$ or 2 lead to

(i ) $\quad m\left(x^{i} y^{j}, x^{k}\right) 1=m\left(x^{i} y^{j} x^{k}, 1\right) 1=m\left(x^{k} y^{j} x^{i}, 1\right) 1$

(ii) $m\left(x^{i} y^{j}, y^{k}\right) 1=m\left(x^{i} y^{k+j}, 1\right) 1=m\left(y^{j+k} x^{i}, 1\right) 1$

(iii) $m\left(x^{i} y^{j}, x^{k} y^{l}\right) 1=m\left(x^{i} y^{j+l} x^{k}, 1\right) 1$

(iv) $m\left(x^{i} y^{j}, y^{k} x^{l}\right) 1=m\left(x^{i} y^{j} x^{l} y^{k}, 1\right) 1$.

For (i) $m\left(x^{i} y^{j}, x^{k}\right) 1=\left\{V_{x^{i}} m\left(y^{j}, x^{k}\right)-m\left(y^{j}, x^{i+k}\right)\right\} 1=x^{i} \circ\left(x^{k} \circ y^{j}\right)-x^{i+k} \circ y^{j}$ is symmetric in $i$ and $k$ since $V_{x^{i}}, V_{x^{k}}$ commute, and

$$
\begin{aligned}
& m\left(x^{i} y^{j} x^{k}, 1\right) 1=\left\{m\left(x^{i} y^{j}, 1\right) V_{x^{k}}-m\left(x^{i} y^{j}, x^{k}\right)\right\} 1 \\
& \quad=2\left\{x^{i} y^{j} x^{k}\right\}-\left\{V_{x^{i}} V_{x^{k}}-V_{x^{i+k}}\right\} y^{j} \\
& =\left\{V_{x^{i}} V_{x^{k}}+V_{x^{k}} V_{x^{i}}-V_{x^{k} x^{i}}\right\} y^{j}-\left\{V_{x^{k}} V_{x^{i}}-V_{x^{k+i}}\right\} y^{j} \\
& =\left\{V_{x^{i}} V_{x^{k}}-V_{x^{i+k}}\right\} y^{j} .
\end{aligned}
$$

Similarly, for (ii) $m\left(x^{i} y^{j+k}, 1\right) 1=x^{i} \circ y^{j+k}=m\left(y^{j+k} x^{i}, 1\right) 1$ and

$$
\begin{aligned}
& m\left(x^{i} y^{j}, y^{k}\right) 1=\left\{m\left(x^{i}, y^{k}\right) V_{y^{j}}-m\left(x^{i}, y^{j+k}\right)\right\} 1 \\
& =2\left\{x^{i} y^{j} y^{k}\right\}-x^{i} \circ y^{j+k} \\
& =\left\{V_{x^{i}} V_{y^{k}}+V_{y^{k}} V_{x^{i}}-V_{x^{i} \circ y^{k}}\right\} y^{j}-x^{i} \circ y^{j+k} \\
& =x^{i} \circ y^{j+k}
\end{aligned}
$$

(we are using the relation $V_{z} V_{w}+V_{w} V_{z}-V_{z \circ w}=2 U_{z, w}$ ). For (iii)

$$
\begin{aligned}
& m\left(x^{i} y^{j}, x^{k} y^{l}\right)-m\left(x^{i} y^{j+l} x^{k}, 1\right) 1 \\
& \quad=V_{x^{i}}\left\{m\left(x^{k} y^{l}, y^{j}\right)-m\left(y^{j+l} x^{k}, 1\right)\right\} 1-\left\{m\left(x^{i+k} y^{l}, y^{j}\right)-m\left(y^{j+l} x^{k}, x^{i}\right)\right\} 1 \\
& \quad=0 \text { (by (ii)) }
\end{aligned}
$$

and for (iv)

$$
\begin{aligned}
m\left(x^{i} y^{j},\right. & \left.y^{k} x^{l}\right)-m\left(x^{i} y^{j} x^{l} y^{k}, 1\right) 1 \\
= & V_{x^{i}}\left\{m\left(y^{k} x^{l}, y^{j}\right)-m\left(y^{j} x^{l} y^{k}, 1\right)\right\} 1-U_{x^{i}, y}\left\{m\left(y^{k} x^{l}, 1\right)-m\left(x^{l} y^{k}, 1\right)\right\} 1 \\
& +\left\{m\left(y^{j+k} x^{l}, x^{i}\right)-m\left(x^{i+l} y^{k}, y^{j}\right)\right\} 1 \\
= & 0 \text { (by (i), (ii)). }
\end{aligned}
$$

Now let $h(p)=1$. If $h(q) \geqq 2$ write $q=r s$ for $h(r), h(s) \geqq 1$, and $p=t w$ for $t=p, w=1$. Then we can repeat the argument (15) using the case $h(p) \geqq 2$ : (A), (B) follow since $h(r s) \geqq 2$, (C) since $h(r t) \geqq 1+1=2$, and (D) since $h\left(t w s^{*}\right) \geqq 1+0+1=2$. The remaining case is $h(p)=h(q)=1$, leading to

$$
\begin{aligned}
& m\left(x^{i}, y^{j}\right) 1=U_{x^{i}, y^{j}} 1=x^{i} \circ y^{j}=V_{x^{i}, y} 1=m\left(x^{i} y^{j}, 1\right) 1 \\
& m\left(x^{i}, x^{j}\right) 1=\left\{V_{x^{i}} V_{x^{j}}-m\left(x^{i+j}, 1\right)\right\} 1=2 x^{i} \circ x^{j}-2 x^{i+j}=m\left(x^{i+j}, 1\right) 1 .
\end{aligned}
$$

Finally, let $h(p)=0$. If $h(q) \geqq 2$ we write $q=r s, p=t w$ as above. Then the argument (15) follows from the case $h(p) \geqq 1$ since in (A), (B) we have $h(r s) \geqq 1+1>1$, in (C) $h(r t) \geqq 1+0=1$, and 
in (D) $h\left(t w s^{*}\right) \geqq 0+0+1=1$. The remaining case $h(q)=1$ is trivial, $m\left(1, x^{i}\right) 1=m\left(x_{i}, 1\right) 1$.

This completes the induction on $h(p)+h(q)$ and the proof of the lemma.

As we have seen, the lemma was all we needed to establish

SHIRSHOV'S THEOREM. The free unital quadratic Jordan algebra $\mathfrak{\Im}^{(2)}$ on two generators is special.

It also allows us to reformulate Macdonald's Theorem in a more conceptual way. Recall [4, p. 000] that a Jordan algebra is strongly special if it and all its quadratic representations are special. In view of version V of Macdonald's Theorem we have the following result, which implies both Shirshov's and Macdonald's Theorems.

MACDONALD'S THEOREM (Conceptual form). The free unital quadratic Jordan algebra $\mathfrak{\Im}^{(2)}$ on two generators is strongly special.

Similar arguments can be used to prove $\mathfrak{\Im}^{(2 / 1)}$ and $\mathfrak{\Im}^{(2 / 2)}$ are strongly special, and in particular we have a Shirshov's Theorem with Inverses.

For quadratic Jordan algebras in general there is no analogue of the Shirshov-Cohn Theorem which states that an arbitrary linear Jordan algebra with two generators is special. Indeed, there is a standard example of a quadratic Jordan algebra with one generator $z$ which is not special since $z^{2}=0$ but $z^{3} \neq 0$.

\section{REFERENCES}

1. P. Cohn, Universal algebra, Harper and Row, New York, 1965.

2. N. Jacobson, Structure and Representations of Jordan Algebras, Colloq. Publ. Vol. 39, Amer. Math. Soc., Providence, R.I., 1968.

3. I. G. Macdonald, Jordan algebras with three generators, Proc. London Math. Soc. 10 (1960), 395-408.

4. K. McCrimmon, Representations of quadratic Jordan algebras, Trans. Amer. Math. Soc. 153 (1971).

5. — Macdonald's theorem with inverses, Pacific J. Math. 21 (1967), 315-325.

6. - A general theory of Jordan rings, Proc. Nat. Acad. Sci. U.S.A. 56 (1966), $1072-1079$.

Received December 10, 1969.

UNIVERSITY OF VIRGINIA

Charlottesville, Virginia 


\section{PACIFIC JOURNAL OF MATHEMATICS}

\section{EDITORS}

\author{
H. SAMELSON \\ Stanford University \\ Stanford, California 94305 \\ Richard Pierce \\ University of Washington \\ Seattle, Washington 98105
}

\author{
J. DugundJI \\ Department of Mathematics \\ University of Southern California \\ Los Angeles, California 90007 \\ RICHARD ARENS \\ University of California \\ Los Angeles, California 9.0024
}

\section{ASSOCIATE EDITORS}

\begin{tabular}{|c|c|}
\hline E. F. BECKENBACH & K. YoshidA \\
\hline \multicolumn{2}{|c|}{ SUPPORTING INSTITUTIONS } \\
\hline UNIVERSITY OF BRITISH COLUMBIA & STANFORD UNIVERSITY \\
\hline CALIFORNIA INSTITUTE OF TECHNOLOGY & UNIVERSITY OF TOKYO \\
\hline UNIVERSITY OF CALIFORNIA & UNIVERSITY OF UTAH \\
\hline MONTANA STATE UNIVERSITY & WASHINGTON STATE UNIVERSITY \\
\hline UNIVERSITY OF NEVADA & UNIVERSITY OF WASHINGTON \\
\hline NEW MEXICO STATE UNIVERSITY & * \\
\hline OREGON STATE UNIVERSITY & AMERICAN MATHEMATICAL SOCIETY \\
\hline UNIVERSITY OF OREGON & CHEVRON RESEARCH CORPORATION \\
\hline OSAKA UNIVERSITY & TRW SYSTEMS \\
\hline UNIVERSITY OF SOUTHERN CALIFORNIA & NAVAL WEAPONS CENTER \\
\hline
\end{tabular}

The Supporting Institutions listed above contribute to the cost of publication of this Journal, but they are not owners or publishers and have no responsibility for its content or policies.

Mathematical papers intended for publication in the Pacific Journal of Mathematics should be in typed form or offset-reproduced, (not dittoed), double spaced with large margins. Underline Greek letters in red, German in green, and script in blue. The first paragraph or two must be capable of being used separately as a synopsis of the entire paper. The editorial "we" must not be used in the synopsis, and items of the bibliography should not be cited there unless absolutely necessary, in which case they must be identified by author and Journal, rather than by item number. Manuscripts, in duplicate if possible, may be sent to any one of the four editors. Please classify according to the scheme of Math. Rev. Index to Vol. 39. All other communications to the editors should be addressed to the managing editor, Richard Arens, University of California, Los Angeles, California, 90024.

50 reprints are provided free for each article; additional copies may be obtained at cost in multiples of 50 .

The Pacific Journal of Mathematics is published monthly. Effective with Volume 16 the price per volume (3 numbers) is $\$ 8.00$; single issues, $\$ 3.00$. Special price for current issues to individual faculty members of supporting institutions and to individual members of the American Mathematical Society: $\$ 4.00$ per volume; single issues $\$ 1.50$. Back numbers are available.

Subscriptions, orders for back numbers, and changes of address should be sent to Pacific Journal of Mathematics, 103 Highland Boulevard, Berkeley, California, 94708.

PUBLISHED BY PACIFIC JOURNAL OF MATHEMATICS, A NON-PROFIT CORPORATION

Printed at Kokusai Bunken Insatsusha (Internatıonal Academic Printing Co., Ltd.), 7-17, Fujimi 2-chome, Chiyoda-ku, Tokyo, Japan. 


\section{Pacific Journal of Mathematics}

\section{Vol. 35, No. $3 \quad$ November, 1970}

John D. Arrison and Michael Rich, On nearly commutative degree one algebras . . . 533

Bruce Alan Barnes, Algebras with minimal left ideals which are Hilbert spaces . . . . 537

Robert F. Brown, An elementary proof of the uniqueness of the fixed point index . . . 549

Ronn L. Carpenter, Principal ideals in F-algebras .................... 559

Chen Chung Chang and Yiannis (John) Nicolas Moschovakis, The Suslin-Kleene

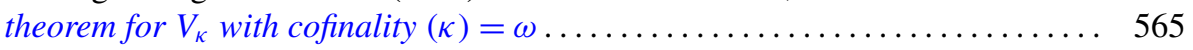

Theodore Seio Chihara, The derived set of the spectrum of a distribution

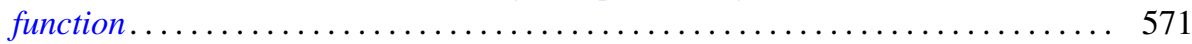

Tae Geun Cho, On the Choquet boundary for a nonclosed subspace of $C(S) \ldots \ldots \quad 575$

Richard Brian Darst, The Lebesgue decomposition, Radon-Nikodym derivative,

conditional expectation, and martingale convergence for lattices of sets .......

David E. Fields, Dimension theory in power series rings . . . . . . . . . . . .

Michael Lawrence Fredman, Congruence formulas obtained by counting

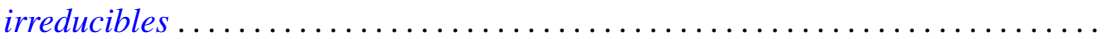

John Eric Gilbert, On the ideal structure of some algebras of analytic functions.....

G. Goss and Giovanni Viglino, Some topological properties weaker than

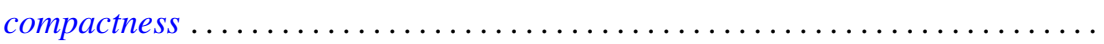

581

601

625

George Grätzer and J. Sichler, On the endomorphism semigroup (and category) of

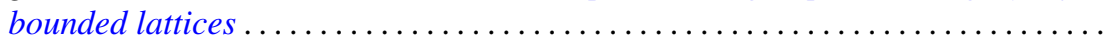

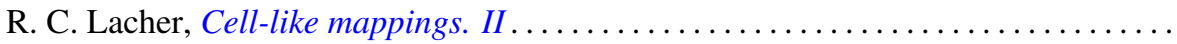

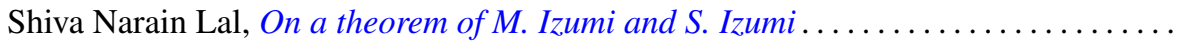

661

Howard Barrow Lambert, Differential mappings on a vector space ...............

Richard G. Levin and Takayuki Tamura, Notes on commutative power joined

semigroups.

Robert Edward Lewand and Kevin Mor McCrimmon, Macdonald's theorem for quadratic Jordan algebras.

J. A. Marti, On some types of completeness in topological vector spaces ....

Walter J. Meyer, Characterization of the Steiner point

717

Saad H. Mohamed, Rings whose homomorphic images are $q$-rings ...

727

Thomas V. O'Brien and William Lawrence Reddy, Each compact orientable surface

of positive genus admits an expansive homeomorphism ...

737

Robert James Plemmons and M. T. West, On the semigroup of binary relations...

743

Calvin R. Putnam, Unbounded inverses of hyponormal operator . .

755

William T. Reid, Some remarks on special disconjugacy criteria for differential

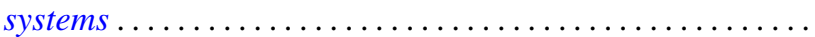

C. Ambrose Rogers, The convex generation of convex Borel sets in euclidean

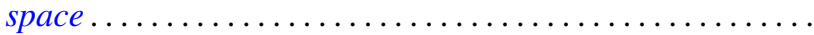

S. Saran, A general theorem for bilinear generating functions .

S. W. Smith, Cone relationships of biorthogonal systems ......

Wolmer Vasconcelos, On commutative endomorphism rings ....

795

Vernon Emil Zander, Products of finitely additive set functions from Orlicz

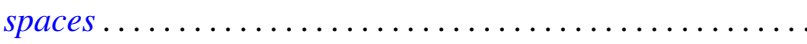

G. Sankaranarayanan and C. Suyambulingom, Correction to: "Some renewal

theorems concerning a sequence of correlated random variables" .

Joseph Zaks, Correction to: "Trivially extending decompositions of $E^{n}$ ”....... 805

Dong Hoon Lee, Correction to: "The adjoint group of Lie groups" ............ 805

James Edward Ward, Correction to: "Two-groups and Jordan algebras". 